\title{
Renewal Theory for Transient Markov Chains with Asymptotically Zero Drift
}

\author{
Denis Denisov, Dmitry Korshunov ${ }^{\dagger}$ and Vitali Wachtel ${ }^{\ddagger}$
}

April 6, 2020

\begin{abstract}
We solve the problem of asymptotic behaviour of the renewal measure (Green function) generated by a transient Lamperti's Markov chain $X_{n}$ in $\mathbb{R}$, that is, when the drift of the chain tends to zero at infinity. Under this setting, the average time spent by $X_{n}$ in the interval $(x, x+1]$ is roughly speaking the reciprocal of the drift and tends to infinity as $x$ grows.

For the first time we present a general approach relying in a diffusion approximation to prove renewal theorems for Markov chains. We apply a martingale type technique and show that the asymptotic behaviour of the renewal measure heavily depends on the rate at which the drift vanishes. The two main cases are distinguished, either the drift of the chain decreases as $1 / x$ or much slower than that, say as $1 / x^{\alpha}$ for some $\alpha \in(0,1)$.

The intuition behind how the renewal measure behaves in these two cases is totally different. While in the first case $X_{n}^{2} / n$ converges weakly to a $\Gamma$ distribution and there is no law of large numbers available, in the second case a strong law of large numbers holds true for $X_{n}^{1+\alpha} / n$ and further normal approximation is available.
\end{abstract}

AMS 2010 subject classifications: Primary 60K05; secondary 60J05; 60G42

Keywords and phrases: Transient Markov chain, renewal kernel, renewal measure, Lamperti's problem, Green function.

\section{Introduction}

Let $X=\left\{X_{n}, n \geq 0\right\}$ be a time homogeneous Markov chain whose state space is some Borel subset $S$ of $\mathbb{R}$, that is, for all $x \in S$ and Borel sets $B \subseteq S$,

$$
\begin{aligned}
\mathbb{P}\left\{X_{n+1} \in B \mid X_{0}, \ldots, X_{n-1}, X_{n}=x\right\} & =\mathbb{P}\left\{X_{n+1} \in B \mid X_{n}=x\right\} \\
=: & P(x, B) .
\end{aligned}
$$

\footnotetext{
*University of Manchester, UK, denis.denisov@manchester.ac.uk

${ }^{\dagger}$ Lancaster University, UK, d.korshunov@lancaster.ac.uk

${ }^{\ddagger}$ University of Augsburg, Germany, vitali.wachtel@math.uni-augsburg.de
} 
Standard examples of $S$ are $\mathbb{R}, \mathbb{Z}, \mathbb{R}^{+}$, and $\mathbb{Z}^{+}$. In the sequel we just say that $X_{n}$ takes values in $\mathbb{R}$ keeping in mind that the corresponding transition probabilities may be defined on some subset $S$ of the real line only.

Denote by $\xi(x), x \in \mathbb{R}$, a random variable corresponding to the jump of the chain at point $x$, that is, a random variable with distribution

$$
\begin{aligned}
\mathbb{P}\{\xi(x) \in B\} & =\mathbb{P}\left\{X_{n+1}-X_{n} \in B \mid X_{n}=x\right\} \\
& =\mathbb{P}_{x}\left\{X_{1} \in x+B\right\}, \quad B \in \mathcal{B}(\mathbb{R})
\end{aligned}
$$

hereinafter the subscript $x$ denotes the initial position of the Markov chain $X_{n}$, that is, $X_{0}=x$. Denote the $k$ th moment of the jump at point $x$ by

$$
m_{k}(x):=\mathbb{E} \xi^{k}(x)
$$

Define the renewal (or potential) kernel $Q$ by the equality

$$
Q(\cdot, \cdot):=\sum_{n=0}^{\infty} P^{n}(\cdot, \cdot) .
$$

A Markov chain $X_{n}$ is called transient (see Meyn and Tweedie [31, Ch. 8]) if there exists a countable cover of $\mathbb{R}$ by uniformly transient sets $\left\{B_{k}\right\}$. In its turn a set $B \in \mathcal{B}(\mathbb{R})$ is called uniformly transient if

$$
\sup _{y \in B} Q(y, B)<\infty .
$$

By the Markov property, this is equivalent to the condition

$$
\sup _{y \in \mathbb{R}} Q(y, B)<\infty
$$

because, considering the first hitting time of $B$, we observe by the Markov property that $Q(x, B) \leq \sup _{y \in B} Q(y, B)$ for all states $x \in \mathbb{R}$. If $X_{n}$ is transient with respect to the collection of intervals $B_{k}=(k, k+1], k \in \mathbb{Z}$, then $Q(x, B)<\infty$ for all $x$ and bounded sets $B$ and, hence, the renewal measure (Green function) generated by the chain $X_{n}$

$$
H(B):=\sum_{n=0}^{\infty} \mathbb{P}\left\{X_{n} \in B\right\}, \quad B \in \mathcal{B}(\mathbb{R}),
$$

is finite for every initial distribution of the chain and bounded set $B$.

The main aim of the present paper is to study integral (elementary) and local renewal theorems for the renewal measure $H$, that is we find asymptotics for $H\left(x_{*}, x\right]$, $H(x, x+t(x)], H(x, x+h]$ as $x \rightarrow \infty$, where $t(x)$ is a growing function and $x_{*}$ and $h$ are some fixed constants.

The simplest case of a transient Markov chain is just a random walk $X_{n}=$ $\xi_{1}+\cdots+\xi_{n}$ generated by independent identically distributed random variables $\xi_{n}$ 's with positive drift, which may be equivalently defined as a spatially and temporarily 
homogeneous Markov chain. The renewal theory for a random walk has been intensively studied since 1940s. The integral (elementary) renewal theorem for a random walk with positive jumps and finite mean goes back to Feller [15] and states that $H(0, x] \sim x / \mathbb{E} \xi_{1}$ as $x \rightarrow \infty$. A more detailed information is available via the local renewal theorem, which was proved for lattice random variables in [13] and for nonlattice random variables in [5]. In the finite mean non-lattice case the local renewal theorem gives the following sharp asymptotics $H(x, x+h] \rightarrow h / \mathbb{E} \xi_{1}$ as $x \rightarrow \infty$, for any fixed $h>0$. Later Blackwell extended in [6] the local renewal theorem to the case of i.i.d. random variables with positive mean that can take values of both signs using the important concept of what was called by Feller ladder heights and ladder epochs. Original Blackwell's proof was considered to be quite complicated and a number of attempts were made to give an easier proof. A rather simple proof was given by Feller and Orey [16, see also [17]. Further studies also considered behaviour of the remainder in the local renewal theorem, see [36] and references therein. In the infinite mean case the asymptotics in Blackwell's theorem was not sharp. In 1960-70s a local renewal theorem was proved for regularly varying increments of in$\operatorname{dex} \alpha>1 / 2$, see [18] and [14. Subsequently there have been various improvements on these results, but the complete answer has been obtained very recently, see [7].

There exists a number of generalisations of the renewal theorem for various stochastic processes. A natural extension is one for non-homogeneous (in time) random walk, that is a random walk with independent, but not necessarily identically distributed increments. Probably the first result in this direction was [9], where the local renewal theorem was derived from the local central limit theorem for a non-homogeneous random walk. Further extensions may be found in [38, 41, 27]. Renewal theorems for multidimensional random walks may be found in [12, [32, [19] and recent paper [3], see also references therein.

The Markov setting has mostly been considered in the literature for case of Markov modulated random walks, see, e.g. 21, 2, 22, and [40]. In this setting one can usually use the Harris regeneration and split the process into independent cycles. Then, the traditional setting of Blackwell's theorem can be used.

For the results cited above, it is essential that the underlying process possesses some independence structure. In the present paper we consider transient Markov chains where the cycle structure is not available, which makes a reduction to Blackwell's theorem impossible. Clearly, in order to observe some regular asymptotics for the renewal process, we need to assume some regular behaviour of the Markov chain at infinity. In particular, if the drift of $X_{n}, m_{1}(x)$, has a positive limit at infinity, say $a$, then the local renewal result, $H(x, x+h] \rightarrow h / a$, is only known for an asymptotically homogeneous in space Markov chain which is defined as a Markov chain such that, for some random variable $\xi$,

$$
\xi(x) \Rightarrow \xi \quad \text { as } x \rightarrow \infty,
$$

see [23]; if there is no asymptotic homogeneity in space then the asymptotic behaviour of $H(x, x+h]$ may be very different.

So, while the asymptotic behaviour of a Markov chain with asymptotically nonzero drift is well understood, at least if it is asymptotically homogeneous, the case 
of a drift vanishing at infinity is studied much less. In general, we say that a Markov chain has asymptotically (in space) zero drift if $m_{1}(x)=\mathbb{E} \xi(x) \rightarrow 0$ as $x \rightarrow \infty$. The study of processes with asymptotically zero drift was initiated by Lamperti in a series of papers [24, 25, 26]. The vanishing drift seems to be more difficult for the analysis due to the fact that the Markov chain tends to infinity much slower and one should take into account diffusion fluctuations.

An integral (elementary) renewal theorem for a transient Markov chain with drift $m_{1}(x)$ asymptotically proportional to $1 / x$ at infinity was proved in [11; it was shown there that then the renewal function behaves as $c x^{2}$ for large values of $x$.

Here we present for the first time a unified approach that allows us to prove renewal theorems for general Markov chains. This is the main novelty of the present paper. In this paper we analyse one-dimensional Markov chains, but clearly the approach suggested below can be used in the multidimensional setting as well. Our approach relies on the diffusion approximation, for that reason we consider Markov chains which may be approximated by diffusion process. Then, if we have some result of renewal type for a diffusion processes we should be able to obtain a similar result for a Markov chain having similar asymptotic behaviour of the first two moments of jumps. In particular, we will see in the examples below that as soon as we have a Green function for the diffusion process we should, in principle, be able to construct an approximation for the Green function of the Markov chain and thus to derive a renewal theorem.

\subsection{Main results on renewal measure}

Throughout we assume some weak irreducibility of $X_{n}$, namely that there are no bounded trajectories of $X_{n}$, that is,

$$
\limsup _{n \rightarrow \infty} X_{n}=\infty \text { a.s. }
$$

For any $s>0$ we denote the $s$-truncation of the $k$ th moment of jump at state $x$ by

$$
m_{k}^{[s]}(x):=\mathbb{E}\left\{\xi^{k}(x) ;|\xi(x)| \leq s\right\} .
$$

For any random variables $\xi$ and $\eta$ we write $\xi \leq_{\text {st }} \eta$ if $\mathbb{P}\{\xi>t\} \leq \mathbb{P}\{\eta>t\}$ for all $t \in \mathbb{R}$.

Theorem 1. Let $X_{n}$ be such that (2) holds and

$$
m_{1}^{[s(x)]}(x) \sim \frac{\mu}{x}, \quad m_{2}^{[s(x)]}(x) \rightarrow b \in(0, \infty) \quad \text { as } x \rightarrow \infty,
$$

for some $\mu>b / 2$ and an increasing level $s(x)$ of order $o(x)$. Assume also that,

$$
\mathbb{P}\{|\xi(y)| \geq s(y)\} \leq p(y) / y,
$$

for some decreasing integrable at infinity function $p(x)$, and

$$
|\xi(y)| \mathbb{I}\{|\xi(y)| \leq s(y)\} \quad \leq_{\text {st }} \quad \widehat{\xi} \quad \text { for all } y \geq 0,
$$


where

$$
\mathbb{E} \widehat{\xi}^{2}<\infty
$$

Then, for every function $h(x) \uparrow \infty$ of order o $(x)$, we have

$$
H(x, x+h(x)] \sim \frac{2}{2 \mu-b} x h(x) \quad \text { as } x \rightarrow \infty .
$$

Notice that the both conditions (44) and (5) are met if $|\xi(y)| \leq_{\text {st }} \xi$ for all $y$ and for some $\xi$ satisfying (6).

In the course of the proof of this and subsequent theorems we construct a bounded non-negative supermartingale, which also shows that $X_{n} \rightarrow \infty$ a.s. This convergence clearly implies that $X_{n}$ is transient. Transience in the case of $\mu>b / 2$ was considered under various additional conditions in a series of papers, see e.g. [24, Theorem 3.1] or [30, Theorem 3.2.3].

Under slightly stronger assumptions, an integral renewal theorem was proved in [11, Theorem 5] where it was shown that $H(0, x] \sim x^{2} /(2 \mu-b)$ as $x \rightarrow \infty$.

We now turn to the critical case $\mu=b / 2$ where the properties of the chainparticularly recurrence and transience - depend on further terms in asymptotic expansions for the moments of increments. As the next theorem shows this is also true for the renewal function.

Theorem 2. Let $X_{n}$ be such that (2) holds and that there exist $m \geq 1, \gamma>0$ and an increasing level $s(x)$ of order $o(x)$ such that

$$
\frac{2 m_{1}^{[s(x)]}(x)}{m_{2}^{[s(x)]}(x)}=\frac{1}{x}+\frac{1}{x \log x}+\ldots+\frac{1}{x \log x \ldots \log _{(m-1)} x}+\frac{\gamma+1+o(1)}{x \log x \ldots \log _{(m)} x}
$$

and $m_{2}^{[s(x)]}(x) \rightarrow b>0$ as $x \rightarrow \infty$. Assume that, for some $\varepsilon>0$,

$$
\begin{array}{rll}
\mathbb{P}\{|\xi(x)|>s(x)\} & = & o\left(1 / x^{2} \log ^{2+\varepsilon} x\right), \\
\mathbb{E}\left\{|\xi(x)|^{3} ;|\xi(x)| \leq s(x)\right\} & = & o\left(x / \log ^{1+\varepsilon} x\right), \\
|\xi(y)| \mathbb{I}\{|\xi(y)| \leq s(y)\} & \text { st }_{\text {st }} & \widehat{\xi},
\end{array}
$$

where $\widehat{\xi}$ satisfies (6). Then, for every function $h(x) \uparrow \infty$ of order o $(x)$, we have

$$
H(x, x+h(x)] \sim \frac{2 h(x)}{b \gamma} x \log x \ldots \log _{(m)} x \quad \text { as } x \rightarrow \infty .
$$

Transience in a similar setting goes back to [29, Theorem 3].

As we have mentioned above, the integral renewal theorem in the case $\mu>b / 2$ was proved in [11]. The proof in that paper is based on the convergence of $X_{n}^{2} / n$ towards $\Gamma$-distribution. This approach is not applicable under the conditions of Theorem 2, although the convergence to $\Gamma$-distribution is still valid. The reason is that some chains with $\mu=b / 2$ are null-recurrent while other are transient, but this difference disappears in the weak limit. The only statement which can be obtained from weak convergence here is the following lower bound:

$$
\lim _{x \rightarrow \infty} \frac{H(0, x]}{x^{2}}=\infty
$$


In the next theorem we consider the case where the drift decreases slower than $1 / x$, that is, $m_{1}(x) x \rightarrow \infty$.

Theorem 3. Let $X_{n}$ satisfy the condition (2) and $v$ be a decreasing function such that $x v(x) \rightarrow \infty$ and $v^{\prime}(x)=o\left(v^{2}(x)\right)$. Let there exist an increasing level $s(x)$ of order o $(1 / v(x))$ such that

$$
m_{1}^{[s(x)]}(x) \sim v(x), \quad m_{2}^{[s(x)]}(x) \rightarrow b \in(0, \infty) \quad \text { as } x \rightarrow \infty,
$$

where $v$ is a decreasing function such that $x v(x) \rightarrow \infty$ and $v^{\prime}(x)=o\left(v^{2}(x)\right)$. Assume also that,

$$
\begin{aligned}
& \mathbb{P}\{|\xi(y)| \geq s(y)\} \leq p(y) v(y), \\
& |\xi(y)| \mathbb{I}\{|\xi(y)|<s(y)\} \leq_{\text {st }} \widehat{\xi} \quad \text { for all } y \geq 0,
\end{aligned}
$$

where $p(x)$ is a non-increasing, non-negative integrable at infinity function, and $\widehat{\xi}$ satisfies (6). Then, for every function $h(x) \uparrow \infty$ of order $o(1 / v(x))$, we have

$$
H(x, x+h(x)] \sim \frac{h(x)}{v(x)} \quad \text { as } x \rightarrow \infty .
$$

In the two examples - nearest neighbour Markov chain and diffusion processconsidered in the two subsections below it is possible to construct an appropriate martingale which allows us to find the renewal measure in a closed form. For general Markov chains considered in the last three theorems, this martingale approach does not work because it is hopeless to construct such a martingale. However, it is possible to construct almost a martingale that allows us to derive the asymptotic behaviour of the renewal measure; it is done in Section 2.

While the asymptotic behaviour of the renewal measure on growing intervals is derived under assumptions on regular behaviour of the first two moments only, it seems that the local renewal theorem can be only proved for asymptotically homogeneous in space Markov chain. The next result gives us a tool for deriving asymptotic behaviour of the renewal measure on intervals from results for sufficiently slowly growing intervals. It requires weak convergence of jumps, see (1).

Theorem 4. Let (11) hold and the family of random variables $\{|\xi(x)|, x \in \mathbb{R}\}$ admit an integrable majorant $\Xi$, that is, $\mathbb{E} \Xi<\infty$ and

$$
|\xi(x)| \leq_{\text {st }} \Xi \text { for all } x \in \mathbb{R} .
$$

Assume that there exist a bounded function $v(x)>0$, a growing level $\widetilde{t}(x) \uparrow \infty$ and a constant $C_{H}<\infty$ such that, for any $t(x) \uparrow \infty$ satisfying $t(x) \leq \widetilde{t}(x)$,

$$
\frac{v(x) H(x, x+t(x)]}{t(x)} \rightarrow C_{H} \quad \text { as } x \rightarrow \infty .
$$

If the limiting random variable $\xi$ is non-lattice, then $v(x) H(x, x+h] \rightarrow C_{H} h$ as $x \rightarrow \infty$, for all fixed $h>0$.

If the chain $X_{n}$ is integer valued and $\mathbb{Z}$ is the minimal lattice for the variable $\xi$, then $v(k) H\{k\} \rightarrow C_{H}$ as $k \rightarrow \infty$. 
Let us apply the last result to chains considered in Theorems 13 ,

Corollary 5. Under the conditions of Theorem 1, (11) and (12), we have, for every $h>0$,

$$
H(x, x+h] \sim \frac{2 h}{2 \mu-b} x \quad \text { as } x \rightarrow \infty,
$$

if the limiting random variable $\xi$ is non-lattice, and

$$
H\{k\} \sim \frac{2}{2 \mu-b} k \quad \text { as } k \rightarrow \infty,
$$

if the chain $X_{n}$ is integer valued and $\mathbb{Z}$ is the minimal lattice for the variable $\xi$.

Corollary 6. Under the conditions of Theorem Q 2, (11) and (12), we have, for every $h>0$,

$$
H(x, x+h] \sim \frac{2 h}{b \gamma} x \log x \ldots \log _{(m)} x \quad \text { as } x \rightarrow \infty,
$$

if the limiting random variable $\xi$ is non-lattice, and

$$
H\{k\} \sim \frac{2}{b \gamma} k \log k \ldots \log _{(m)} k \quad \text { as } k \rightarrow \infty,
$$

if the chain $X_{n}$ is integer valued and $\mathbb{Z}$ is the minimal lattice for the variable $\xi$.

Corollary 7. Under the conditions of Theorem [3, (11) and (12), we have, for every $h>0$,

$$
H(x, x+h] \sim \frac{h}{v(x)} \text { as } x \rightarrow \infty,
$$

if the limiting random variable $\xi$ is non-lattice, and

$$
H\{k\} \sim \frac{1}{v(k)} \text { as } k \rightarrow \infty,
$$

if the chain $X_{n}$ is integer valued and $\mathbb{Z}$ is the minimal lattice for the variable $\xi$.

Markov chains with asymptotically zero drift naturally appear in various areas including branching processes, stochastic difference equations, networks, etc. In most cases we are aware of it gives rise to the drift of order $O(1 / x)$ at infinity. We now consider the random walk conditioned to stay positive, which represents one of the classical examples of chains with asymptotically zero drift.

Let $S_{n}$ be a random walk with independent identically distributed increments $\xi_{k}$, that is, $S_{n}=\xi_{1}+\xi_{2}+\ldots+\xi_{n}, n \geq 1$. Let $\tau_{x}$ be the first time when $S_{n}$ started at $x$ is non-positive:

$$
\tau_{x}:=\min \left\{n \geq 1: x+S_{n} \leq 0\right\}
$$


We assume that the random walk $S_{n}$ is oscillating, that is,

$$
\liminf _{n \rightarrow \infty} X_{n}=-\infty, \quad \limsup _{n \rightarrow \infty} X_{n}=\infty \quad \text { with probability } 1 .
$$

In particular, $\mathbb{P}\left\{\tau_{x}<\infty\right\}=1$ for all starting points $x$. Let $\chi^{-}$denote the first weak descending ladder height of $S_{n}$, that is, $\chi^{-}=-S_{\tau_{0}}$. Let $V(x)$ denote the renewal function corresponding to weak descending ladder heights of our random walk:

$$
V(x):=1+\sum_{k=1}^{\infty} \mathbb{P}\left\{\chi_{1}^{-}+\chi_{2}^{-}+\ldots+\chi_{k}^{-}<x\right\},
$$

where $\chi_{k}^{-}$are independent copies of $\chi^{-}$.

It is well-known - see e.g. Bertoin and Doney [4] - that $V(x)$ is a harmonic function for $S_{n}$ killed at leaving $(0, \infty)$. More precisely,

$$
V(x)=\mathbb{E}\left\{V\left(x+S_{1}\right) ; \tau_{x}>1\right\}, \quad x \geq 0 .
$$

This implies that Doob's $h$-transform

$$
P(x, d y):=\frac{V(y)}{V(x)} \mathbb{P}\left\{x+S_{1} \in d y, \tau_{x}>1\right\}
$$

defines a stochastic transition kernel on $\mathbb{R}^{+}$. Let $X_{n}$ be the corresponding Markov chain, which we shall call random walk conditioned to stay positive.

Example 8. Let $\mathbb{E} \xi_{1}=0$ and $\sigma^{2}:=\mathbb{E} \xi_{1}^{2} \in(0, \infty)$. Then the renewal measure $H$ of the random walk conditioned to stay positive has the following asymptotic behaviour: for every fixed $h>0$,

$$
H(x, x+h] \sim \frac{2 h}{\sigma^{2}} x \quad \text { as } x \rightarrow \infty
$$

if $\xi_{1}$ is non-lattice, and

$$
H\{k\} \sim \frac{2}{\sigma^{2}} k \quad \text { as } k \rightarrow \infty, k \in \mathbb{Z},
$$

if $\mathbb{Z}$ is the minimal lattice for $\xi_{1}$.

In Section 6, we provide a proof based on Corollary 5, It is worth mentioning that the finiteness of $\mathbb{E} \xi_{1}^{2}$ does not imply existence of second moments of $X_{n}$. Thus, this example underlines the advantage of our assumptions in terms of truncated moments. Let us note that one can also prove the last result making use of Proposition 19.3 from Spitzer [39] in lattice case and Port and Stone 34 in non-lattice case, together with the well-known result on the renewal measures of the descending and ascending ladder height processes associated with the random walk.

For Markov chains considered in Theorems 1 and 2 one knows that $X_{n}^{2} / n$ converges in distribution towards a $\Gamma$-distribution. Since this convergence takes place without centering, $X_{n}$ tends to infinity diffusively slow. The influence of the diffusion component is expressed by the fact that the renewal function grows at rate 
$2 x /(2 \mu-b)$ which is strictly greater than the reciprocal of the drift at point $x$. The chains satisfying the conditions of Theorem 3 go to infinity much faster, and a law of large numbers holds. More precisely, if the drift is of order $x^{-\alpha}, \alpha \in(0,1)$ then $X_{n}^{1+\alpha} / n$ converges to a positive constant. As a result, we have the classical form of the local renewal theorem: the rate of growth is asymptotically equivalent to the reciprocal of the drift. We believe that Theorem 3 remains valid for chains with unbounded second moments, but we do not know how to prove it.

We conclude this section by noting that Markov chains with growing second moments can be transformed sometimes to chains with bounded second moments. First we consider a critical branching process with immigration. Let $\left\{\zeta_{n, k}\right\}_{n, k \geq 1}$ be independent copies of a random variable $\zeta$ valued in $\mathbb{Z}^{+}$. Assume that $\mathbb{E} \zeta=1$ and $\sigma^{2}:=\mathbb{E} \zeta^{2} \in(0, \infty)$. Let $\left\{\eta_{n}\right\}_{n \geq 1}$ be i.i.d. random variables which are also independent of $\left\{\zeta_{n, k}\right\}$. Assume that $a:=\mathbb{E} \eta_{1}>0$ and $\mathbb{E} \eta_{1}^{2}<\infty$. Consider the Markov chain

$$
Z_{n+1}=\sum_{k=1}^{Z_{n}} \zeta_{n+1, k}+\eta_{n+1}, \quad n \geq 0 .
$$

For this chain one has

$$
\begin{aligned}
\mathbb{E}\left\{Z_{1}-Z_{0} \mid Z_{0}=k\right\} & =a, \\
\mathbb{E}\left\{\left(Z_{1}-Z_{0}\right)^{2} \mid Z_{0}=k\right\} & =\sigma^{2} k+\mathbb{E} \eta_{1}^{2} .
\end{aligned}
$$

Since the second moments of increments are linearly growing we cannot apply our results directly to this chain. However one can consider the chain $X_{n}=\sqrt{Z_{n}}$, which then satisfies the conditions of Theorem 1 with $\mu=\left(a-\sigma^{2} / 4\right) / 2$ and $b=\sigma^{2} / 4$. Furthermore, the central limit theorem implies that $X_{n}$ is asymptotically homogeneous in space and the limiting variable $\xi$ is normally distributed with parameters 0 and $\sigma^{2} / 4$. Then, applying Corollary 5 , we obtain the local renewal theorem for $X_{n}$. Performing the inverse transformation, one gets asymptotics for the renewal function of $Z_{n}$ on the intervals $[k, k+h \sqrt{k})$. Unfortunately, our approach does not allow us to consider shorter intervals. An integral renewal theorem for $Z_{n}$ has been obtained by Pakes [33], while Mellein [28] has proved the corresponding local renewal theorem. Their proofs use the machinery of generating functions.

In general, if the second moments of jumps of $X_{n}$ are growing as $x^{\beta}, \beta \in(0,2)$, then the jumps of $X_{n}^{1-\beta / 2}$ have bounded second moments and one can try to apply one of our theorems.

\subsection{Key renewal theorem}

We now turn to the renewal equation

$$
Z(B)=z(B)+\int_{\mathbb{R}} Z(d y) P(y, B), \quad B \in \mathcal{B}(\mathbb{R}),
$$

where $z$ is a finite non-negative measure on $\mathbb{R}$. This is more than sufficient to ensure that

$$
Z(B)=\int_{\mathbb{R}} z(d u) H_{u}(B), \quad B \in \mathcal{B}(\mathbb{R}),
$$


is a unique locally finite solution to the renewal equation. The analysis of the preceding subsection of this paper allows us to deduce the asymptotic behaviour of the measure $Z$ at infinity. The proof is immediate from the dominated convergence theorem.

Theorem 9. Let $B \in \mathcal{B}(\mathbb{R})$. Assume that, for some positive function $g(x)$ and for all $y \in \mathbb{R}$,

$$
H_{y}(x+B) \sim g(x) \text { as } x \rightarrow \infty,
$$

and, for some $c<\infty$,

$$
H_{y}(x+B) \leq c g(x) \quad \text { for all } x, y \in \mathbb{R} .
$$

If $z$ is a finite measure, then

$$
Z(x+B) \sim z(\mathbb{R}) g(x) \quad \text { as } x \rightarrow \infty .
$$

\subsection{Nearest-neighbour Markov chain}

To illustrate the approach and intuition beyond the results above, we consider first a nearest-neighbour (skip-free or continuous) Markov chain $X_{n}$ on $\mathbb{Z}^{+}$, that is, $\xi(x)$ only takes values $-1,1$ and 0 , with probabilities $p_{-}(x), p_{+}(x)$ and $p_{0}(x)=1-$ $p_{-}(x)-p_{+}(x)$ respectively, $p_{-}(0)=0$. Nearest-neighbour Markov chains are very useful for our purposes because in this case one can write down an expression for the renewal measure in a closed form and then analyse it.

For a nearest-neighbour Markov chain $X_{n}$ with specific jump probabilities, $p_{-}(x)=$ $(1-\lambda /(x+\lambda)) / 2$ and $p_{+}(x)=(1+\lambda /(x+\lambda)) / 2, \lambda>-1 / 2$ (which corresponds to transience of $X_{n}$ ), Guivarc'h et al. [20, Theorems 42 and 43] have obtained weak convergence of $X_{n}^{2} / n$ to the $\Gamma_{\lambda+1 / 2,2}$-distribution and the local renewal theorem in that case. They used the technique of orthogonal polynomials, in Rosenkrantz [37, which is only available for specific Markov chains considered in that paper.

Let

$$
p_{+}(k)=p+\varepsilon_{+}(k) \quad \text { and } \quad p_{-}(k)=p-\varepsilon_{-}(k), \quad p \leq 1 / 2 .
$$

Assume that $\varepsilon_{ \pm}(k) \rightarrow 0$ as $k \rightarrow \infty$ that is the case of asymptotically zero drift and convergent second moment of jumps, $m_{2}(k) \rightarrow 2 p$ as $k \rightarrow \infty$.

We can define the renewal measure (Green function) of $X_{n}$ as follows

$$
h_{x_{0}}(x):=\sum_{n=0}^{\infty} \mathbb{P}_{x_{0}}\left\{X_{n}=x\right\}, \quad x_{0}, x \in \mathbb{Z}^{+} .
$$

Since we consider a Markov chain with jumps $-1,1$ and 0 only, $h_{x_{0}}(x)=h_{x}(x)$ for all $x_{0} \leq x$. Below we demonstrate how to find $h_{x_{0}}(x)$ in a closed form.

We first look for a function $g(x, z) \geq 0$ such that, for all $x$, the process

$$
W_{n}=g\left(x, X_{n}\right)-\sum_{k=0}^{n-1} \mathbb{I}\left\{X_{k}=x\right\}
$$


is a martingale which happens if $g$ satisfies the following system of equations

$$
\begin{aligned}
& g(x, 0)=p_{0}(0) g(x, 0)+p_{+}(0) g(x, 1), \\
& g(x, y)=p_{-}(y) g(x, y-1)+p_{0}(y) g(x, y)+p_{+}(y) g(x, y+1)-\mathbb{I}\{y=x\}, \quad y \geq 1 .
\end{aligned}
$$

Take $g(x, 0)=g(x, 1)=\ldots=g(x, x)=0$. Then for $y=x$ we get

$$
g(x, x+1)-g(x, x)=g(x, x+1)=\frac{1}{p_{+}(x)}=\frac{1}{p_{-}(x)} \frac{p_{-}(x)}{p_{+}(x)},
$$

and, for $y \geq x+1$,

$$
\begin{aligned}
g(x, y+1)-g(x, y) & =\frac{p_{-}(y)}{p_{+}(y)}(g(x, y)-g(x, y-1)) \\
& =\prod_{z=x+1}^{y} \frac{p_{-}(z)}{p_{+}(z)}(g(x, x+1)-g(x, x)) \\
& =\frac{1}{p_{+}(x)} \prod_{z=x+1}^{y} \frac{p_{-}(z)}{p_{+}(z)} \\
& =\frac{1}{p_{-}(x)} \prod_{z=x}^{y} \frac{p_{-}(z)}{p_{+}(z)} .
\end{aligned}
$$

Therefore, for $y \geq x+1$,

$$
\begin{aligned}
g(x, y)=\sum_{u=x}^{y-1}(g(x, u+1)-g(x, u)) & =\frac{1}{p_{+}(x)} \sum_{u=x}^{y-1} \prod_{z=x+1}^{u} \frac{p_{-}(z)}{p_{+}(z)} \\
& =\frac{1}{p_{-}(x)} \sum_{u=x}^{y-1} \prod_{z=x}^{u} \frac{p_{-}(z)}{p_{+}(z)}
\end{aligned}
$$

which is increasing in $y$. This sequence is bounded provided

$$
\sum_{u=1}^{\infty} \prod_{z=1}^{u} \frac{p_{-}(z)}{p_{+}(z)}<\infty
$$

Then denote

$$
g(x, \infty):=\lim _{y \rightarrow \infty} g(x, y)=\frac{1}{p_{+}(x)} \sum_{u=x}^{\infty} \prod_{z=x+1}^{u} \frac{p_{-}(z)}{p_{+}(z)} .
$$

The sequence (15) is a martingale, so for all $n$,

$$
g\left(x, x_{0}\right)=\mathbb{E}_{x_{0}} W_{0}=\mathbb{E}_{x_{0}} W_{n}=\mathbb{E}_{x_{0}} g\left(x, X_{n}\right)-\mathbb{E}_{x_{0}} \sum_{k=0}^{n-1} \mathbb{I}\left\{X_{k}=x\right\}
$$

and hence

$$
\sum_{k=0}^{n-1} \mathbb{P}_{x_{0}}\left\{X_{k}=x\right\}=\mathbb{E}_{x_{0}} g\left(x, X_{n}\right)-g\left(x, x_{0}\right)<g(x, \infty)<\infty .
$$


Finiteness of the renewal measure implies the transience of $X_{n}$, hence $X_{n} \rightarrow \infty$ as $n \rightarrow \infty$ a.s. Thus, we get the following explicit representation for the renewal measure

$$
\begin{aligned}
h_{x_{0}}(x)=g(x, \infty)-g\left(x, x_{0}\right) & =\frac{1}{p_{+}(x)} \sum_{u=x \vee x_{0}}^{\infty} \prod_{z=x+1}^{u} \frac{p_{-}(z)}{p_{+}(z)} \\
& =\frac{1}{p_{-}(x)} \sum_{u=x \vee x_{0}}^{\infty} \prod_{z=x}^{u} \frac{p_{-}(z)}{p_{+}(z)} \\
& =\frac{1}{p_{+}(x)} \prod_{z=1}^{x} \frac{p_{+}(z)}{p_{-}(z)} \sum_{u=x \vee x_{0}}^{\infty} \prod_{z=1}^{u} \frac{p_{-}(z)}{p_{+}(z)} .
\end{aligned}
$$

We have

$$
\prod_{z=x}^{u} \frac{p_{-}(z)}{p_{+}(z)}=\exp \left\{\sum_{z=x}^{u} \log \frac{1-\varepsilon_{-}(z) / p}{1+\varepsilon_{+}(z) / p}\right\} .
$$

Assume that

$$
\frac{2 m_{1}(x)}{m_{2}(x)} \sim r(x) \quad \text { as } x \rightarrow \infty
$$

where $r(x)$ is a differentiable decreasing function such that the quotient $r^{\prime}(x) / r^{2}(x)$ has a limit at infinity. The last asymptotic equivalence is equivalent to

$$
\log \frac{1-\varepsilon_{-}(x) / p}{1+\varepsilon_{+}(x) / p} \sim-r(x) \quad \text { as } x \rightarrow \infty .
$$

Fix an $\varepsilon>0$. Then for all sufficiently large $x$ we can write

$$
-(1+\varepsilon) r(x) \leq \log \frac{1-\varepsilon_{-}(x) / p}{1+\varepsilon_{+}(x) / p} \leq-(1-\varepsilon) r(x) .
$$

Therefore, for such $x$, we have the following upper bound

$$
\begin{aligned}
h_{x_{0}}(x) & \leq \frac{1}{p_{-}(x)} \sum_{u=x}^{\infty} \exp \left\{-(1-\varepsilon) \sum_{z=x}^{u} r(z)\right\} \\
& \leq \frac{1}{p_{-}(x)} \int_{x}^{\infty} \exp \left\{-(1-\varepsilon) \int_{x}^{u} r(z) d z\right\} d u
\end{aligned}
$$

due to the decrease of $r(z)$. Putting

$$
U_{\varepsilon}(x)=\int_{x}^{\infty} \exp \left\{-(1-\varepsilon) \int_{0}^{u} r(z) d z\right\} d u
$$

we observe that

$$
\int_{x}^{\infty} \exp \left\{-(1-\varepsilon) \int_{x}^{u} r(z) d z\right\} d u=\frac{U_{\varepsilon}(x)}{-U_{\varepsilon}^{\prime}(x)}
$$


By L'Hospital's rule,

$$
\begin{aligned}
\lim _{x \rightarrow \infty} \frac{U_{\varepsilon}(x)}{-U_{\varepsilon}^{\prime}(x) / r(x)} & =\lim _{x \rightarrow \infty} \frac{U_{\varepsilon}^{\prime}(x)}{-U_{\varepsilon}^{\prime \prime}(x) / r(x)+U_{\varepsilon}^{\prime}(x) r^{\prime}(x) / r^{2}(x)} \\
& =\frac{1}{1-\varepsilon+\lim _{x \rightarrow \infty} r^{\prime}(x) / r^{2}(x)} .
\end{aligned}
$$

Therefore,

$$
\limsup _{x \rightarrow \infty} h_{x_{0}}(x) r(x) \leq \frac{1}{p} \frac{1}{1-\varepsilon+\lim _{x \rightarrow \infty} r^{\prime}(x) / r^{2}(x)}
$$

Similarly, starting from inequalities

$$
\begin{aligned}
h_{x_{0}}(x) & \geq \frac{1}{p_{+}(x)} \sum_{u=x}^{\infty} \exp \left\{-(1+\varepsilon) \sum_{z=x+1}^{u} r(z)\right\} \\
& \geq \frac{1}{p_{+}(x)} \int_{x}^{\infty} \exp \left\{-(1-\varepsilon) \int_{x}^{u} r(z) d z\right\} d u
\end{aligned}
$$

we get a lower bound

$$
\liminf _{x \rightarrow \infty} h_{x_{0}}(x) r(x) \geq \frac{1}{p} \frac{1}{1+\varepsilon+\lim _{x \rightarrow \infty} r^{\prime}(x) / r^{2}(x)} .
$$

Since $\varepsilon>0$ is arbitrary we conclude that

$$
h_{x_{0}}(x) \sim \frac{1}{p r(x)} \frac{1}{1+\lim _{y \rightarrow \infty} r^{\prime}(y) / r^{2}(y)} \quad \text { as } x \rightarrow \infty .
$$

In the following two examples we consider canonical drifts where $r^{\prime}(y) / r^{2}(y)$ has either negative or zero limit at infinity.

Example 10. If $\varepsilon_{+}(k) \sim \mu_{+} / k$ and $\varepsilon_{-}(k) \sim \mu_{-} / k$ as $k \rightarrow \infty$ and $\mu:=\mu_{+}+\mu_{-}>p$, then (18) is valid with $r(x)=\mu / p x, r^{\prime}(x) / r^{2}(x) \rightarrow-p / \mu$, and we deduce that

$$
h_{x_{0}}(x) \sim \frac{x}{\mu-p} \text { as } x \rightarrow \infty .
$$

Example 11. If $\varepsilon_{+}(k) \sim \mu_{+} / k^{\alpha}$ and $\varepsilon_{-}(k) \sim \mu_{-} / k^{\alpha}$ as $k \rightarrow \infty, \mu:=\mu_{+}+\mu_{-}>0$, $\alpha \in(0,1)$, then (18) is valid with $r(x)=\mu / p x^{\alpha}, r^{\prime}(x) / r^{2}(x) \rightarrow 0$, and we deduce $a$ Weibullian asymptotics for the renewal measure at infinity,

$$
h_{x_{0}}(x) \sim \frac{x^{\alpha}}{\mu} \sim \frac{1}{m_{1}(x)} \quad \text { as } x \rightarrow \infty .
$$

Let us note that a lower bound in Example 10 may be deduced from the local limit theorem from Alexander [1, Theorem 2.4]. 


\subsection{Diffusion process}

Now let us consider another Markov process allowing solutions in closed form, a transient diffusion $X_{t}$ on $\mathbb{R}$ (or $\mathbb{R}^{+}$) with the following generator

$$
A=\mu(x) \frac{d}{d x}+\frac{\sigma^{2}(x)}{2} \frac{d^{2}}{d x^{2}} .
$$

We consider a regular diffusion, in the sense of properties (i)-(iii) of [35, Chapter VII.3]. For the transience it is sufficient to assume that the following function

$$
U(x):=\int_{x}^{\infty} \exp \left\{-\int_{0}^{v} \frac{2 \mu(y)}{\sigma^{2}(y)} d y\right\} d v
$$

is finite for all $x$. This function solves the homogeneous equation

$$
A U=0 .
$$

In this case $X_{t} \rightarrow \infty$ a.s. and we are interested in the continuous time analogue of the renewal function,

$$
H_{y}(x, x+h]:=\int_{0}^{\infty} \mathbb{P}_{y}\left\{X_{t} \in(x, x+h]\right\} d t .
$$

It is known that the process $f\left(X_{t}\right)-f\left(X_{0}\right)-\int_{0}^{t} A f\left(X_{s}\right) d s$ is a local martingale. Fix $x$ and $h$. Suppose we can find a bounded function $f(z)=f_{h, x}(z)$ such that $f(z) \rightarrow 0$ as $z \rightarrow \infty$ and

$$
A f(z)=-\mathbb{I}\{z \in(x, x+h]\} .
$$

Then the optional stopping theorem and a.s. convergence $X_{t} \rightarrow \infty$ as $t \rightarrow \infty$ will give us an equality

$$
f(y)=\mathbb{E}_{y} f\left(X_{0}\right)=\mathbb{E}_{y}\left[\int_{0}^{\infty} \mathbb{I}\left\{X_{t} \in(x, x+h]\right\} d t\right]=H_{y}(x, x+h],
$$

which allows us to analyse $H_{y}$.

So, we need to solve the ordinary differential equation (21). To this end, consider

$$
m(x):=\int_{0}^{x} \frac{2 d v}{-U^{\prime}(v) \sigma^{2}(v)}=\int_{0}^{x} \frac{2}{\sigma^{2}(v)} \exp \left\{\int_{0}^{v} \frac{2 \mu(y)}{\sigma^{2}(y)} d y\right\} d v
$$

and then

$$
G_{x}(z):= \begin{cases}U(z) m(x), & z \geq x \\ U(z) m(z)+\int_{z}^{x} U(v) m(d v), & z<x .\end{cases}
$$

We have

$$
\frac{d}{d z} G_{x}(z)= \begin{cases}U^{\prime}(z) m(x), & z \geq x \\ U^{\prime}(z) m(z), & z<x\end{cases}
$$


and

$$
\frac{d^{2}}{d z^{2}} G_{x}(z)= \begin{cases}U^{\prime \prime}(z) m(x), & z \geq x, \\ U^{\prime \prime}(z) m(z)-2 / \sigma^{2}(z), & z<x\end{cases}
$$

which together with (20) implies that

$$
A G_{x}(z)= \begin{cases}-1, & z \leq x \\ 0, & z>x\end{cases}
$$

and hence the function

$$
f(z)=G_{h, x}(z):=G_{x+h}(z)-G_{x}(z)
$$

solves (21). Alternatively one can notice that $U(x)$ corresponds to the scale function and $m(x)$ to the speed measure and that (see [35, Chapter VII, Theorem 3.12])

$$
A G_{x}(z)=\frac{d}{d m(z)}\left(\frac{d G_{x}(z)}{-d U(z)}\right)
$$

Thus, if follows from (22) that for $y<x$,

$$
H_{y}(x, x+h]=\int_{x}^{x+h} U(v) m(d v)=\int_{x}^{x+h} \frac{2 U(v) d v}{-U^{\prime}(v) \sigma^{2}(v)} .
$$

More formally one can obtain the last equality from Corollary 3.8 and Exercise 3.20 in [35, Ch. VII.3].

If the function $W(v):=U(v) / U^{\prime}(v) \sigma^{2}(v)$ is long tailed at infinity-that is, for any fixed $u, W(v+u) \sim W(v)$ as $v \rightarrow \infty$ - then we get the following local renewal theorem for $X_{t}$ starting at $y$,

$$
H_{y}(x, x+h] \sim \frac{2 U(x)}{-U^{\prime}(x) \sigma^{2}(x)} h \quad \text { as } x \rightarrow \infty .
$$

Assume that

$$
2 \mu(x) / \sigma^{2}(x) \sim r(x) \text { as } x \rightarrow \infty,
$$

for some differentiable function $r(x)$ such that the quotient $r^{\prime}(x) / r^{2}(x)$ has a limit at infinity. Hence, we can apply L'Hospital's rule to obtain

$$
\begin{aligned}
\lim _{x \rightarrow \infty} \frac{U(x)}{-U^{\prime}(x) / r(x)} & =\lim _{x \rightarrow \infty} \frac{U^{\prime}(x)}{-U^{\prime \prime}(x) / r(x)+U^{\prime}(x) r^{\prime}(x) / r^{2}(x)} \\
& =\frac{1}{1+\lim _{x \rightarrow \infty} r^{\prime}(x) / r^{2}(x)} .
\end{aligned}
$$

Therefore, for any fixed $h>0$,

$$
H_{y}(x, x+h] \sim \frac{2}{\sigma^{2}(x) r(x)} \frac{1}{1+\lim _{y \rightarrow \infty} r^{\prime}(y) / r^{2}(y)} h \quad \text { as } x \rightarrow \infty .
$$


Note that this asymptotics do not assume existence of the limit of the variance $\sigma^{2}(x)$ at infinity, and that happens because of very specific nature of diffusion processes compared to Markov chains. In order to get a result for Markov chains with growing second truncated moment of jumps, one would definitely need to assume regular growth of that moments at infinity. It is also clear that convergence of Markov chains to a stable law will play a rôle then.

Similar to nearest neighbour Markov chains, in the following two examples we consider canonical drifts where $r^{\prime}(y) / r^{2}(y)$ has either negative or zero limit at infinity.

Example 12. If $\mu(x) \sim \mu / x$ and $\sigma^{2}(x) \rightarrow \sigma^{2}>0$ as $x \rightarrow \infty$ with $2 \mu>\sigma^{2}$, then (23) is satisfied with $r(x)=2 \mu / \sigma^{2} x, r^{\prime}(x) / r^{2}(x) \rightarrow-\sigma^{2} / 2 \mu$, and we get

$$
H_{y}(x, x+h] \sim \frac{2 h}{2 \mu-\sigma^{2}} x \quad \text { as } x \rightarrow \infty .
$$

Example 13. If $\mu(x) \sim \mu / x^{\alpha}, \mu>0, \alpha \in(0,1)$, and $\sigma^{2}(x) \rightarrow \sigma^{2}>0$ as $x \rightarrow \infty$, then (23) is satisfied with $r(x)=2 \mu / \sigma^{2} x^{\alpha}, r^{\prime}(x) / r^{2}(x) \rightarrow 0$, and we get

$$
H_{y}(x, x+h] \sim \frac{h}{\mu} x^{\alpha} \sim \frac{h}{\mu(x)} \text { as } x \rightarrow \infty .
$$

\section{Preliminary bounds for renewal measure on growing intervals}

Let $h(x)$ be an unboundedly growing function. This section is mostly devoted to the construction of functions $G_{h, x}^{*}(y)$ and $G_{h, x}^{* *}(y)$ such that the processes $G_{h, x}^{*}\left(X_{n}\right)$ and $G_{h, x}^{* *}\left(X_{n}\right)$ have drifts, roughly speaking, not less and not greater than the limiting jump variance times $\mathbb{I}\{y \in[x, x+h(x)]\}$ respectively. That allows us to conclude upper and lower bounds for the renewal measure on the interval $[x, x+h(x)]$ of growing length.

Let $r(x)$ be a decreasing differentiable function on $[0, \infty)$ satisfying the condition

$$
r^{\prime}(x)=O\left(r^{2}(x)\right) \text { as } x \rightarrow \infty,
$$

in the sequel $r(x)$ approximates the quotient $2 m_{1}^{[s(x)]}(x) / m_{2}^{[s(x)]}(x)$. We shall impose assumptions on the truncated moments of Markov chains, and doing that we always assume that the truncation function $s(x)$ increases and satisfies

$$
s(x)=o(1 / r(x)) \quad \text { as } x \rightarrow \infty .
$$

Define

$$
R(z):=\int_{0}^{z} r(y) d y, \quad U(x):=\int_{x}^{\infty} e^{-R(z)} d z,
$$

where $U(x)$ is assumed finite, compare to $U$ defined in (19). Clearly,

$$
\frac{U^{\prime \prime}(y)}{U^{\prime}(y)}=-r(y)
$$


Let us fix an increasing function $s(x)$ of order $o(1 / r(x))$ as $x \rightarrow \infty$. Due to (24),

$$
r(x+y) \sim r(x), \quad R(x+y)-R(x) \rightarrow 0 \quad \text { and } \quad e^{-R(x+y)} \sim e^{-R(x)}
$$

as $x \rightarrow \infty$ uniformly for $|y| \leq s(x)$. Also,

$$
U^{\prime \prime \prime}(x)=\left(r^{2}(x)-r^{\prime}(x)\right) e^{-R(x)}=O\left(r^{2}(x) e^{-R(x)}\right)
$$

and, consequently,

$$
U^{\prime \prime \prime}(x+y)=O\left(r^{2}(x) e^{-R(x)}\right) \quad \text { as } x \rightarrow \infty \text { uniformly for }|y| \leq s(x) .
$$

Let

$$
G(y):=U(0)-U(y)=\int_{0}^{y} e^{-R(z)} d z .
$$

We start with a result showing that $G\left(X_{n}\right)$ is almost a martingale provided the quotient $2 m_{1}^{[s(x)]}(x) / m_{2}^{[s(x)]}(x)$ is asymptotically proportional to $r(x)$.

Lemma 14. Let $\theta(y)$ be a non-negative bounded function. Let

$$
\mathbb{E}\left\{|\xi(y)|^{3} ;|\xi(y)| \leq s(y)\right\}=o\left(m_{2}^{[s(y)]}(y) \theta(y) / r(y)\right) \quad \text { as } y \rightarrow \infty .
$$

(i) If

$$
\mathbb{P}\{\xi(y)<-s(y)\}=0 \quad \text { for all } y \geq 0
$$

and

$$
\frac{2 m_{1}^{[s(y)]}(y)}{m_{2}^{[s(y)]}(y)} \geq(1+\theta(y)) r(y) \text { for all sufficiently large } y,
$$

then there exists a $y^{*}>0$ such that

$$
\mathbb{E}\{G(y+\xi(y))-G(y) ; \xi(y) \leq s(y)\} \geq 0 \quad \text { for all } y>y^{*} .
$$

(ii) If

$$
\mathbb{P}\{\xi(y)>s(y)\}=0 \quad \text { for all } y \geq 0
$$

and

$$
\frac{2 m_{1}^{[s(y)]}(y)}{m_{2}^{[s(y)]}(y)} \leq(1-\theta(y)) r(y) \quad \text { for all sufficiently large } y,
$$

then there exists a $y^{*}>0$ such that

$$
\mathbb{E}\{G(y+\xi(y))-G(y) ; \xi(y) \geq-s(y)\} \leq 0 \quad \text { for all } y>y^{*} .
$$


Proof. (i) Since the function $G(y)$ is increasing,

$$
\mathbb{E} G(y+\xi(y))-G(y) \geq \mathbb{E}\{G(y+\xi(y))-G(y) ;|\xi(y)| \leq s(y)\},
$$

due to the condition (30). Since $G^{\prime}(y)=e^{-R(y)}, G^{\prime \prime}(y)=-r(y) e^{-R(y)}$, and $G^{\prime \prime \prime}(y+$ $z)=O\left(r^{2}(y)\right) e^{-R(y)}$ as $y \rightarrow \infty$ uniformly for all $|z| \leq s(y)$ due to the upper bound (28) on $U^{\prime \prime \prime}$ and (26) , application of Taylor's expansion up to the third derivative yields that, for some $\gamma=\gamma(x, \xi(x)) \in[0,1]$,

$$
\begin{aligned}
\mathbb{E}\{G(y+\xi(y))-G(y) ; & |\xi(y)| \leq s(y)\} \\
=m_{1}^{[s(y)]}(y) G^{\prime}(y)+ & \frac{1}{2} m_{2}^{[s(y)]}(y) G^{\prime \prime}(y) \\
& +\frac{1}{6} \mathbb{E}\left\{\xi^{3}(y) G^{\prime \prime \prime}(y+\gamma \xi(y)) ;|\xi(y)| \leq s(y)\right\} \\
=m_{1}^{[s(y)]}(y) e^{-R(y)}- & \frac{1}{2} m_{2}^{[s(y)]}(y) r(y) e^{-R(y)} \\
& +O\left(r^{2}(y) e^{-R(y)} \mathbb{E}\left\{\left|\xi^{3}(y)\right| ;|\xi(y)| \leq s(y)\right\}\right) \quad \text { as } y \rightarrow \infty .
\end{aligned}
$$

The sum of the first two terms on the right hand side equals

$$
\frac{1}{2} e^{-R(y)}\left(2 m_{1}^{[s(y)]}(y)-m_{2}^{[s(y)]}(y) r(y)\right) \geq \frac{1}{2} e^{-R(y)} m_{2}^{[s(y)]}(y) \theta(y) r(y),
$$

due to the condition (31). The third term on the right hand side of the previous equation is of order $o\left(m_{2}^{[s(y)]}(y) \theta(y) r(y) e^{-R(y)}\right)$ owing to the condition (29). These observations conclude the proof of (i).

(ii) Since the function $G(y)$ is increasing,

$$
\mathbb{E} G(y+\xi(y))-G(y) \leq \mathbb{E}\{G(y+\xi(y))-G(y) ;|\xi(y)| \leq s(y)\},
$$

due to the condition (32). The rest of the proof is very similar to part (i).

\subsection{Upper bound}

Our derivation of an upper bound for the renewal measure of $X_{n}$ is based on the Lyapunov function $G_{h, x}^{* *}(y)$ defined below in (36).

For any $x$ and $h>0$, consider a piecewise differentiable function

$$
g_{h, x}^{* *}(y):= \begin{cases}0, & y \leq x, \\ 2(y-x), & y \in(x, x+h], \\ 2 h, & y \in(x+h, x+h+s(x+h)], \\ 2 h e^{R(x+h+s(x+h))-R(y)}, & y>x+h+s(x+h),\end{cases}
$$

whose derivative satisfies

$$
g_{h, x}^{* * \prime}(y)=2 \mathbb{I}\{y \in[x, x+h]\} \quad \text { for all } y<x+h+s(x+h), y \neq x, x+h .
$$


Its integral - the function which originates from the key function (22) for diffusion processes,

$$
G_{h, x}^{* *}(y):=\int_{0}^{y} g_{h, x}^{* *}(z) d z,
$$

is an increasing bounded function, $G_{h, x}^{* *}(\infty)<\infty$, because

$$
g_{h, x}^{* *}(y) \leq 2 h e^{R(x+h+s(x+h))-R(y)} \quad \text { for all } y,
$$

and hence,

$$
\begin{aligned}
G_{h, x}^{* *}(\infty) & \leq 2 h \int_{x}^{\infty} e^{R(x+h+s(x+h))-R(y)} d y \\
& =2 h e^{R(x+h+s(x+h))} U(x) \\
& \leq 2 h U(x) e^{R(x+h)+r(x+h) s(x+h)}
\end{aligned}
$$

because $R$ is concave. As $s(x)=o(1 / r(x))$,

$$
\begin{aligned}
G_{h, x}^{* *}(\infty) & \leq 2 h U(x) e^{R(x+h)+o(1)} \\
& \leq 2 h U(x) e^{R(x)+o(1)} \quad \text { as } x \rightarrow \infty,
\end{aligned}
$$

for $h \leq s(x)$, due to (26).

The function $G_{h, x}^{* *}(y)$ is convex for $y \leq x+h$. For $y>x+h$, the function $G_{h, x}^{* *}(y)$ increases in a concave way with slope $2 h$ at point $x+h$. Notice that, for $y>x+h+s(x+h)$ and $z>0$,

$$
G_{h, x}^{* *}(y+z)-G_{h, x}^{* *}(y)=2 h e^{R(x+h+s(x+h))}(G(y+z)-G(y))
$$

and, due to (37), for $y>x+h+s(x+h)$ and $z \leq 0$,

$$
G_{h, x}^{* *}(y+z)-G_{h, x}^{* *}(y) \geq 2 h e^{R(x+h+s(x+h))}(G(y+z)-G(y)) .
$$

Therefore, for all $y>x+h+s(x+h)$ and $z \in \mathbb{R}$

$$
G_{h, x}^{* *}(y+z)-G_{h, x}^{* *}(y) \geq 2 h e^{R(x+h+s(x+h))}(G(y+z)-G(y)) .
$$

Further, for $y \in(x+h, x+h+s(x+h)]$,

$$
g_{h, x}^{* *}(y+z) \geq 2 h e^{R(y)-R(y+z)} \quad \text { for } z>0,
$$

and

$$
g_{h, x}^{* *}(y+z) \leq 2 h e^{R(y)-R(y+z)} \quad \text { for } z \leq 0 .
$$

Therefore, for $y \in(x+h, x+h+s(x+h)]$,

$$
G_{h, x}^{* *}(y+z)-G_{h, x}^{* *}(y) \geq 2 h e^{R(y)}(G(y+z)-G(y)) .
$$

Lemma 15. Assume that the conditions (29) -(31) hold. Then there exists an $x^{*}>0$ such that, for all $x>x^{*}, y \in \mathbb{R}, h \leq s(x)$, and $t \in(0, h / 2)$,

$$
\mathbb{E} G_{h, x}^{* *}(y+\xi(y))-G_{h, x}^{* *}(y) \geq m_{2}^{[t]}(y) \mathbb{I}\{y \in[x+t, x+h-t]\} .
$$


Proof. Since the function $G_{h, x}^{* *}(y)$ is zero for $y \leq x$ and positive for $y>x$, the mean drift of $G_{h, x}^{* *}$ is non-negative for all $y \leq x$ and the inequality (42) follows for this range of $y$.

Since $G_{h, x}^{* *}(y)$ is increasing and due to (30),

$$
\mathbb{E} G_{h, x}^{* *}(y+\xi(y))-G_{h, x}^{* *}(y) \geq \mathbb{E}\left\{G_{h, x}^{* *}(y+\xi(y))-G_{h, x}^{* *}(y) ;|\xi(y)| \leq s(y)\right\}=: E .
$$

Positiveness of $E$ for $y>x+h$ follows from (40) and (41), by Lemma 14

Thus, it remains to estimate $E$ from below for $y \in[x, x+h]$. By Taylor's expansion for $G_{h, x}^{* *}$ with integral remainder term,

$$
E=m_{1}^{[s(y)]}(y) g_{h, x}^{* *}(y)+\mathbb{E}\left\{\int_{y}^{y+\xi(y)} g_{h, x}^{* * \prime}(z)(y+\xi(y)-z) d z ;|\xi(y)| \leq s(y)\right\} .
$$

Since $g_{h, x}^{* *}(z) \geq 0$ and $g_{h, x}^{* * \prime}(z) \geq 0$ for all $z \in[0, x+h+s(x+h)]$, we obtain for all sufficiently large $x$ and $y \in[x, x+h]$

$$
\begin{aligned}
E & \geq \mathbb{E}\left\{\int_{y}^{y+\xi(y)} g_{h, x}^{* * \prime}(z)(y+\xi(y)-z) d z ;|\xi(y)| \leq t\right\} \\
& \geq 2 \mathbb{I}\{y \in[x+t, x+h-t]\} \mathbb{E}\left\{\int_{y}^{y+\xi(y)}(y+\xi(y)-z) d z ;|\xi(y)| \leq t\right\} \\
& =m_{2}^{[t]}(y) \mathbb{I}\{y \in[x+t, x+h-t]\},
\end{aligned}
$$

because $g_{h, x}^{* * \prime}(z)=2$ for all $z \in(x, x+h]$ which concludes the proof.

Proposition 16. Assume that conditions of Lemma 15 hold. Then there exists an $x^{*}>0$ such that, for all $x>x^{*}, h \leq s(x)$, and $t \in(0, h / 2)$,

$$
H(x+t, x+h-t] \leq \frac{G_{h, x}^{* *}(\infty)-\mathbb{E} G_{h, x}^{* *}\left(X_{0}\right)}{\min _{y \in[x+t, x+h-t]} m_{2}^{[t]}(y)} .
$$

Proof. Consider the following decomposition

$$
G_{h, x}^{* *}\left(X_{n}\right)=\sum_{k=0}^{n-1}\left(G_{h, x}^{* *}\left(X_{k+1}\right)-G_{h, x}^{* *}\left(X_{k}\right)\right)+G_{h, x}^{* *}\left(X_{0}\right) .
$$

Since $G_{h, x}^{* *}(y)$ is bounded by $G_{h, x}^{* *}(\infty)$, we obtain

$$
\begin{aligned}
G_{h, x}^{* *}(\infty) & \geq \mathbb{E} G_{h, x}^{* *}\left(X_{n}\right) \\
& =\mathbb{E} G_{h, x}^{* *}\left(X_{0}\right)+\sum_{k=0}^{n-1} \mathbb{E}\left[G_{h, x}^{* *}\left(X_{k+1}\right)-G_{h, x}^{* *}\left(X_{k}\right)\right] \\
& \geq \mathbb{E} G_{h, x}^{* *}\left(X_{0}\right)+\sum_{k=0}^{n-1} \mathbb{E}\left\{m_{2}^{[t]}\left(X_{k}\right) ; X_{k} \in(x+t, x+h-t]\right\},
\end{aligned}
$$


for $x>x_{*}$, by Lemma 15, Hence, for any $n$,

$$
\sum_{k=0}^{n-1} \mathbb{P}\left\{X_{k} \in(x+t, x+h-t]\right\} \leq \frac{G_{h, x}^{* *}(\infty)-\mathbb{E} G_{h, x}^{* *}\left(X_{0}\right)}{\min _{y \in[x+t, x+h-t]} m_{2}^{[t]}(y)} .
$$

Letting $n$ to infinity we arrive at the conclusion.

\subsection{Lower bound}

We now turn to an accompanying lower bound for the renewal measure. To this end we consider a differentiable function

$$
g_{h, x}^{*}(y):= \begin{cases}0, & y \leq x \\ 2(y-x), & y \in(x, x+h], \\ 2 h e^{R(x+h)-R(y)}, & y>x+h\end{cases}
$$

whose derivative satisfies

$$
g_{h, x}^{* \prime}(y) \leq 2 \mathbb{I}\{y \in[x, x+h]\} \quad \text { for all } y \geq 0 .
$$

Its integral - which similar to (36) originates from the key function (22) for diffusion processes,

$$
G_{h, x}^{*}(y):=\int_{0}^{y} g_{h, x}^{*}(z) d z,
$$

is an increasing bounded function, $G_{h, x}^{*}(\infty)<\infty$, and

$$
\begin{aligned}
G_{h, x}^{*}(\infty) & =h^{2}+2 h e^{R(x+h)} U(x+h) \\
& \geq 2 h e^{R(x)} U(x+h) .
\end{aligned}
$$

For $h \leq s(x)=o(1 / r(x))$,

$$
G_{h, x}^{*}(\infty) \geq(2+o(1)) h e^{R(x)} U(x) \quad \text { as } x \rightarrow \infty .
$$

Also define a concave function

$$
G_{h, x}^{*<}(y):=h^{2}+2 h e^{R(x+h)} \int_{x+h}^{y} e^{-R(z)} d z .
$$

Observe the inequality

$$
G_{h, x}^{*}(y) \geq G_{h, x}^{*<}(y) \quad \text { for all } y \leq x+h,
$$

and equality

$$
G_{h, x}^{*}(y)=G_{h, x}^{*<}(y) \text { for all } y>x+h,
$$

Hence, for $y>x+h$ and $z>0$,

$$
\begin{aligned}
G_{h, x}^{*}(y-z)-G_{h, x}^{*<}(y-z) & \leq G_{h, x}^{*}(y)-G_{h, x}^{*<}(y-z) \\
& =G_{h, x}^{*<}(y)-G_{h, x}^{*<}(y-z) \\
& =2 h e^{R(x+h)}(G(y)-G(y-z)) .
\end{aligned}
$$


Lemma 17. Assume that the conditions (29), (32) and (33) hold. Then there exists an $x^{*}>0$ such that, for all $x>x^{*}, y \geq 0, h \leq s(x)$, and $t \in(0, h / 2)$,

$$
\begin{aligned}
& \mathbb{E} G_{h, x}^{*}(y+\xi(y))-G_{h, x}^{*}(y) \\
& \leq \begin{cases}0, & y \leq x-s(x), \\
2 h \mathbb{E}\{\xi(y) ; \xi(y) \in(x-y, s(y))\}, & y \in(x-s(x), x-t], \\
(1+h r(y)) m_{2}^{[s(y)]}(y), & y \in(x-t, x+h+t], \\
3 h \mathbb{E}\{|\xi(y)| ;-s(y)<\xi(y)<x+h-y\}, & y>x+h+t .\end{cases}
\end{aligned}
$$

Proof. Since $G_{h, x}^{*}(y)$ is increasing in $y$, we obtain

$$
\begin{aligned}
\mathbb{E} G_{h, x}^{*}(y+\xi(y))-G_{h, x}^{*}(y) & \leq \mathbb{E}\left\{G_{h, x}^{*}(y+\xi(y))-G_{h, x}^{*}(y) ; \xi(y) \geq-s(y)\right\} \\
& =\mathbb{E}\left\{G_{h, x}^{*}(y+\xi(y))-G_{h, x}^{*}(y) ;|\xi(y)| \leq s(y)\right\}=: E,
\end{aligned}
$$

due to (32).

Case $y \leq x-t$. It follows from the definition of $G_{h, x}^{*}$ that $G_{h, x}^{*}(x+z) \leq 2 h z$ for all $z>0$ which yields $G_{h, x}^{*}(y+z) \leq 2 h(y-x+z)$ for all $y \leq x$ and $z>0$. Therefore,

$$
E \leq 2 h \mathbb{E}\{\xi(y) ; \xi(y) \in(x-y, s(y)]\},
$$

and the conclusion of the lemma follows for $y \leq x-t$.

Case $y \in(x-t, x+h+t]$. We proceed similarly to Lemma [15. By Taylor's expansion (43),

$$
\begin{aligned}
E & \leq m_{1}^{[s(y)]}(y) g_{h, x}^{*}(y)+m_{2}^{[s(y)]}(y) \\
& \leq \frac{1}{2} m_{2}^{[s(y)]}(y) r(y) g_{h, x}^{*}(y)+m_{2}^{[s(y)]}(y) \\
& \leq m_{2}^{[s(y)]}(y)(h r(y)+1),
\end{aligned}
$$

due to (33), (45) and inequality $g_{h, x}^{*}(y) \leq 2 h$, for all sufficiently large $y$. Thus the conclusion of the lemma follows for $y \in(x-t, x+h+t]$.

Case $y>x+h+t$. Since the function $G(y)$ is concave,

$$
G(y-z)-G(y) \leq z G^{\prime}(y-z)=z e^{-R(y-z)} \quad \text { for all } z>0 .
$$

Therefore, as $y \rightarrow \infty$,

$$
G(y-z)-G(y) \leq z e^{-R(y)}(1+o(1)) \quad \text { uniformly for all } z \in[0, s(y)] .
$$

Thus it follows from (52) that, as $y \rightarrow \infty$,

$$
\begin{aligned}
G_{h, x}^{*}(y-z)-G_{h, x}^{*<}(y-z) & \leq 2 h z e^{R(x+h)-R(y)}(1+o(1)) \\
& \leq 2 h z(1+o(1)) \quad \text { uniformly for all } h, z \in[0, s(y)] .
\end{aligned}
$$


The inequality (50) and equality (51) allow us to conclude that, for $y>x+h$,

$$
\begin{aligned}
E= & \mathbb{E}\left\{G_{h, x}^{*<}(y+\xi(y))-G_{h, x}^{*<}(y) ;|\xi(y)| \leq s(y)\right\} \\
& +\mathbb{E}\left\{G_{h, x}^{*}(y+\xi(y))-G_{h, x}^{*<}(y+\xi(y)) ;|\xi(y)| \leq s(y)\right\} \\
= & \mathbb{E}\left\{G_{h, x}^{*<}(y+\xi(y))-G_{h, x}^{*<}(y) ;|\xi(y)| \leq s(y)\right\} \\
& +\mathbb{E}\left\{G_{h, x}^{*}(y+\xi(y))-G_{h, x}^{*<}(y+\xi(y)) ; \xi(y) \in[-s(y), x+h-y]\right\} \\
\leq & \mathbb{E}\left\{G_{h, x}^{*}(y+\xi(y))-G_{h, x}^{*<}(y+\xi(y)) ; \xi(y) \in[-s(y), x+h-y]\right\},
\end{aligned}
$$

by the second statement of Lemma 14. Applying here (54) we deduce, for all sufficiently large $x$ and $y>x+h$,

$$
E \leq 3 h \mathbb{E}\{|\xi(y)| ; \xi(y) \in[-s(y), x+h-y]\} .
$$

Combining altogether we conclude the result of the lemma for $y>x+h+t$.

Proposition 18. Let the assumptions of Lemma 17 hold. Then there exists an $x^{*}>0$ such that, for all $x>x^{*}, y \geq 0, h \leq s(x)$, and $t \in(0, h / 2)$,

$$
H(x-t, x+h+t] \geq \frac{G_{h, x}^{*}(\infty)-\mathbb{E} G_{h, x}^{*}\left(X_{0}\right)-\delta(x)}{\max _{y \in[x-t, x+h+t]}(1+h r(y)) m_{2}^{[s(y)]}(y)},
$$

where

$$
\begin{aligned}
\delta(x)=2 h & \int_{x-s(x)}^{x-t} H(d y) \mathbb{E}\{\xi(y) ; x-y<\xi(y)<s(y)\} \\
& +3 h \int_{x+h+t}^{\infty} H(d y) \mathbb{E}\{|\xi(y)| ;-s(y)<\xi(y)<x+h-y\} .
\end{aligned}
$$

Proof. Consider the decomposition

$$
G_{h, x}^{*}\left(X_{n}\right)=\sum_{k=0}^{n-1}\left(G_{h, x}^{*}\left(X_{k+1}\right)-G_{h, x}^{*}\left(X_{k}\right)\right)+G_{h, x}^{*}\left(X_{0}\right) .
$$

We deduce from Lemma 17 that, for some $c<\infty$ and all $x>x_{*}$,

$$
\begin{aligned}
& \mathbb{E} G_{h, x}^{*}\left(X_{n}\right) \\
& =\mathbb{E} G_{h, x}^{*}\left(X_{0}\right)+\sum_{k=0}^{n-1} \mathbb{E}\left(G_{h, x}^{*}\left(X_{k+1}\right)-G_{h, x}^{*}\left(X_{k}\right)\right) \\
& \leq \mathbb{E} G_{h, x}^{*}\left(X_{0}\right)+\sum_{k=0}^{n-1} \mathbb{E}\left\{\left(1+h r\left(X_{k}\right)\right) m_{2}^{\left[s\left(X_{k}\right)\right]}\left(X_{k}\right) ; X_{k} \in(x-t, x+h+t]\right\} \\
& \quad+2 h \sum_{k=0}^{n-1} \int_{x-s(x)}^{x-t} \mathbb{P}\left\{X_{k} \in d y\right\} \mathbb{E}\{\xi(y) ; x-y<\xi(y)<s(y)\} \\
& \quad+3 h \sum_{k=0}^{n-1} \int_{x+h+t}^{\infty} \mathbb{P}\left\{X_{k} \in d y\right\} \mathbb{E}\{|\xi(y)| ;-s(y)<\xi(y)<x+h-y\} .
\end{aligned}
$$


Hence, for any $n$,

$$
\sum_{k=0}^{n-1} \mathbb{P}\left\{X_{k} \in(x-t, x+h+t]\right\} \geq \frac{\mathbb{E} G_{h, x}^{*}\left(X_{n}\right)-\mathbb{E} G_{h, x}^{*}\left(X_{0}\right)-\delta(x)}{\max _{y \in[x-t, x+h+t]}(1+h r(y)) m_{2}^{[s(y)]}(y)} .
$$

Letting $n$ to infinity we arrive at the conclusion due to the convergence $G_{h, x}^{*}\left(X_{n}\right) \rightarrow$ $G_{h, x}^{*}(\infty)$ which in its turn follows from Lemma 15 together with the martingale convergence theorem and the assumption (2).

In order to get a lower bound in a closed form, we need to derive conditions under which the term $\delta(x)$ in Proposition 18 is of order $o\left(G_{h, x}^{*}(\infty)\right)$ as $x \rightarrow \infty$. In the next result we demonstrate how to bound $\delta(x)$ provided an appropriate upper bound for the renewal measure is available.

Lemma 19. Let

$$
H(x+t, x+h-t] \leq C_{1} h U(x) e^{R(x)} \quad \text { for some } C_{1}<\infty,
$$

and, for some random variable $\xi$ with $\mathbb{E} \xi^{2}<\infty$,

$$
|\xi(y)| \leq_{s t} \quad \xi \quad \text { for all } y \geq 0 .
$$

Then $\delta(x)=o\left(h U(x) e^{R(x)}\right)$ as $x \rightarrow \infty$.

Proof. Let us analyse the first term in $\delta(x)$. The stochastic majorisation condition (56) yields that

$$
\int_{x-s(x)}^{x-t} H(d y) \mathbb{E}\{\xi(y) ; x-y<\xi(y)<s(y)\} \leq \int_{x-s(x)}^{x-t} H(d y) \mathbb{E}\{\xi ; \xi>x-y\} .
$$

Further, using the upper bound (55) applied to $h(x)=3 t$ we deduce

$$
\begin{aligned}
\int_{x-s(x)}^{x-t(x)} H(d y) \mathbb{E}\{\xi ; \xi>x-y\} & \leq \sum_{n=1}^{s(x) / t} H(x-(n+1) t, x-n t] \mathbb{E}\{\xi ; \xi>n t\} \\
& \leq C_{2} t U^{*}(x) e^{R^{*}(x)} \sum_{n=1}^{s(x) / t} \mathbb{E}\{\xi ; \xi>n t\} \\
& \leq C_{2} t U^{*}(x) e^{R^{*}(x)} \mathbb{E}\left\{\xi^{2} / t ; \xi>t\right\} \\
& =o\left(U^{*}(x) e^{R^{*}(x)}\right) \text { as } t, x \rightarrow \infty,
\end{aligned}
$$

by the condition $\mathbb{E} \xi^{2}<\infty$. Hence the first term in $\delta(x)$ is of order $o\left(h(x) U^{*}(x) e^{R^{*}(x)}\right)$ as required. The second term in $\delta(x)$ is of the same order, as follows by the same arguments, and we conclude the proof. 


\section{$3 \quad$ On two Markov chains with asymptotically equal jumps}

In this section, we prove a coupling that allows us to compare two Markov chains which have asymptotically equal jumps. The following result is repeatedly used in the sequel each time we want to simplify our calculations related to the characteristics of $X_{n}$. We formulate this result in the following general setting.

Let $Y_{n}$ and $Z_{n}$ be two Markov chains with jumps $\eta(x)$ and $\zeta(x)$ respectively. Denote by $H_{y}^{Y}$ the renewal measure generated by the chain $Y_{n}$ with initial state $Y_{0}=y$, that is,

$$
H_{y}^{Y}(A):=\sum_{n=0}^{\infty} \mathbb{P}_{y}\left\{Y_{n} \in A\right\}, \quad A \in \mathcal{B}(\mathbb{R}) .
$$

Lemma 20. Let the random variables $\eta(x)$ and $\zeta(x)$ be constructed on the same probability space in such a way that

$$
\mathbb{P}\{\eta(x) \neq \zeta(x)\} \leq p(x) v(x) \text { for all } x,
$$

where $v(x)>0$ and $p(x)>0$ are decreasing functions and $p(x)>0$ is integrable at infinity. Let also, for some $c<\infty$,

$$
H_{y}^{Y}(x, 2 x] \leq \frac{c x}{v(x)} \text { for all } y \text { and } x .
$$

Then, for any $\varepsilon>0$ there exists an $x_{\varepsilon}$ such that the chains $Y_{n}$ and $Z_{n}$ may be constructed on the same probability space in such a way that

$$
\mathbb{P}\left\{Y_{n}=Z_{n} \text { for all } n \geq 0\right\} \geq 1-\varepsilon
$$

provided $Z_{0}=Y_{0}>x_{\varepsilon}$.

Proof. It follows from the condition (58) that, for all $z \in \mathbb{R}$,

$$
\mathbb{P}\left\{Y_{n}>z \text { for all } n \geq 0 \mid Y_{0}=y\right\} \quad \rightarrow \quad 1 \text { as } y \rightarrow \infty .
$$

Let us construct a probability space and two sequences of independent random fields $\left\{\eta_{n}(x), x \in \mathbb{R}\right\}_{n \geq 0}$ and $\left\{\zeta_{n}(x), x \in \mathbb{R}\right\}_{n \geq 0}$ on this space such that

$$
\mathbb{P}\left\{\eta_{n}(x) \neq \zeta_{n}(x)\right\} \leq p(x) v(x) \text { for all } x \in \mathbb{R} \text { and } n \geq 0,
$$

which is possible due to (57). Then let us define Markov chains as follows:

$$
Y_{n+1}=Y_{n}+\eta_{n+1}\left(Y_{n}\right), \quad Z_{n+1}=Z_{n}+\zeta_{n+1}\left(Z_{n}\right),
$$

Fix an $\varepsilon>0$. For any $z$,

$$
\begin{aligned}
& \mathbb{P}\left\{Z_{n} \neq Y_{n} \text { for some } n \mid Y_{0}=y\right\} \\
& \leq \mathbb{P}\left\{Y_{n} \leq z \text { for some } n \mid Y_{0}=y\right\} \\
& \quad+\mathbb{P}\left\{Z_{n} \neq Y_{n} \text { for some } n, Y_{n} \geq z \text { for all } n \mid Y_{0}=y\right\}
\end{aligned}
$$


Owing to (59), there exists an $y_{1}(z)$ such that

$$
\mathbb{P}\left\{Y_{n} \leq z \text { for some } n \mid Y_{0}=y\right\} \leq \varepsilon / 2 \text { for all } y>y_{1}(z) .
$$

Given $Z_{0}=Y_{0}>z$,

$$
\begin{aligned}
\mathbb{P}\left\{Z_{n}\right. & \left.\neq Y_{n} \text { for some } n, Y_{n}>z \text { for all } n \mid Y_{0}=y\right\} \\
& \leq \mathbb{P}\left\{\eta_{n+1}\left(Y_{n}\right) \neq \zeta_{n+1}\left(Z_{n}\right), Y_{n}=Z_{n} \text { for some } n, Y_{n}>z \text { for all } n \mid Y_{0}=y\right\}
\end{aligned}
$$

The probability on the right hand side does not exceed the following sum

$$
\begin{aligned}
\sum_{n=0}^{\infty} \mathbb{P}\left\{\eta_{n+1}\left(Y_{n}\right) \neq\right. & \left.\zeta_{n+1}\left(Z_{n}\right), Z_{n}=Y_{n}>z \mid Y_{0}=y\right\} \\
& \leq \int_{z}^{\infty} \mathbb{P}\{\eta(x) \neq \zeta(x)\} H_{y}^{Y}(d x) \\
& \leq \int_{z}^{\infty} p(x) v(x) H_{y}^{Y}(d x),
\end{aligned}
$$

by the condition (57). The last integral tends to 0 as $z \rightarrow \infty$. Indeed, both functions $p(z)$ and $v(x)$ are decreasing, hence

$$
\int_{2 z}^{\infty} p(x) v(x) H_{y}^{Y}(d x) \leq \sum_{i=1}^{\infty} p\left(x_{i}\right) v\left(x_{i}\right) H_{y}^{Y}\left(x_{i}, x_{i+1}\right],
$$

where $x_{i}:=2^{i-1} z$ for $i \geq 0$. Then, by the condition (58) on $H_{y}^{Y}$,

$$
\begin{aligned}
\int_{2 z}^{\infty} p(x) v(x) H_{y}^{Y}(d x) & \leq c \sum_{i=1}^{\infty} p\left(x_{i}\right) x_{i} \\
& =2 c \sum_{i=1}^{\infty} p\left(x_{i}\right)\left(x_{i}-x_{i-1}\right)
\end{aligned}
$$

Then decrease of the function $p(x)$ yields

$$
\sum_{i=1}^{\infty} p\left(x_{i}\right)\left(x_{i}-x_{i-1}\right) \leq \int_{z}^{\infty} p(u) d u \rightarrow 0 \quad \text { as } z \rightarrow \infty
$$

because $p(x)$ is integrable. Hence,

$$
\int_{2 z}^{\infty} p(x) v(x) H_{y}^{Y}(d x) \rightarrow 0 \quad \text { as } z \rightarrow \infty \text { uniformly for all } y,
$$

which implies convergence to 0 of the integral from $z$ to $\infty$. Then the integral from $z$ to $\infty$ is less than $\varepsilon / 2$ for a sufficiently large $z=z(\varepsilon)$ which concludes the proof with $x_{\varepsilon}=y_{1}(z(\varepsilon))$. 
Lemma 21. Let the conditions of Lemma 20 hold. If there exist non-negative functions $h(x)$ and $g(x)$ such that

$$
H^{Y}(x, x+h(x)] \sim g(x) \quad \text { as } x \rightarrow \infty
$$

for any distribution of $Y_{0}$ and

$$
\sup _{y} H_{y}^{Y}(x, x+h(x)]=O(g(x)) \quad \text { as } x \rightarrow \infty,
$$

then, for any distribution of $Z_{0}$,

$$
H^{Z}(x, x+h(x)] \sim g(x) \quad \text { as } x \rightarrow \infty .
$$

Proof. Let us construct $\left\{\eta_{n}(x), x \in \mathbb{R}\right\}_{n \geq 0}$ and $\left\{\zeta_{n}(x), x \in \mathbb{R}\right\}_{n \geq 0}$ as in (60) and then the Markov chains $Y_{n}$ and $Z_{n}$ as there.

Fix an $\varepsilon>0$ and let $x_{\varepsilon}$ be delivered by the last lemma. Let $\tau:=\min \{n \geq 0$ : $\left.Z_{n}>x_{\varepsilon}\right\}$ and consider $Y_{k}$ with initial value $Y_{0}=Z_{\tau}$. Define

$$
\mu:=\min \left\{k \geq 1: Y_{k} \neq Z_{\tau+k}\right\} .
$$

By Lemma 20, $\mathbb{P}\{\mu<\infty\} \leq \varepsilon$. For $x>x_{\varepsilon}$,

$$
\begin{aligned}
& \sup _{y} H_{y}^{Z}(x, x+h(x)] \\
& \leq \sup _{y} \mathbb{E}_{y} \sum_{n=\tau}^{\tau+\mu-1} \mathbb{I}\left\{Z_{n} \in(x, x+h(x)]\right\}+\sup _{y} \mathbb{E}_{y} \sum_{n=\tau+\mu}^{\infty} \mathbb{I}\left\{Z_{n} \in(x, x+h(x)]\right\} .
\end{aligned}
$$

The first expectation on the right hand side is not greater than $H_{y}^{Y}(x, x+h(x)$ ] because $Z_{n}=Y_{n-\tau}$ between $\tau$ and $\tau+\mu-1$. The second one possesses the following upper bound

$$
\begin{aligned}
\mathbb{E}_{y} \sum_{n=\mu}^{\infty} \mathbb{I}\left\{Z_{n} \in(x, x+h(x)]\right\} & =\mathbb{E}_{y}\left\{\sum_{n=\tau+\mu}^{\infty} \mathbb{I}\left\{Z_{n} \in(x, x+h(x)]\right\} \mid \mu<\infty\right\} \mathbb{P}\{\mu<\infty\} \\
& \leq \sup _{z} H_{z}^{Z}(x, x+h(x)] \varepsilon .
\end{aligned}
$$

Therefore,

$$
\sup _{y} H_{y}^{Z}(x, x+h(x)] \leq \frac{1}{1-\varepsilon} \sup _{y} H_{y}^{Y}(x, x+h(x)]
$$

which, due to assumption (63) implies that

$$
\sup _{y} H_{y}^{Z}(x, x+h(x)]=O(g(x)) .
$$


For any distribution of $Z_{0}$ we have

$$
\begin{aligned}
& H^{Z}(x, x+h(x)] \\
& =\mathbb{E} \sum_{n=\tau}^{\tau+\mu-1} \mathbb{I}\left\{Z_{n} \in(x, x+h(x)]\right\}+\mathbb{E} \sum_{n=\tau+\mu}^{\infty} \mathbb{I}\left\{Z_{n} \in(x, x+h(x)]\right\} \\
& =\mathbb{E} \sum_{n=\tau}^{\tau+\mu-1} \mathbb{I}\left\{Y_{n} \in(x, x+h(x)]\right\}+\mathbb{E} \sum_{n=\tau+\mu}^{\infty} \mathbb{I}\left\{Z_{n} \in(x, x+h(x)]\right\} \\
& =\mathbb{E} H_{Z_{\tau}}^{Y}(x, x+h(x)] \\
& \quad-\quad \mathbb{E} \mathbb{E}_{Z_{\tau}} \sum_{n=\mu}^{\infty} \mathbb{I}\left\{Y_{n} \in(x, x+h(x)]\right\}+\mathbb{E} \sum_{n=\tau+\mu}^{\infty} \mathbb{I}\left\{Z_{n} \in(x, x+h(x)]\right\}
\end{aligned}
$$

According to (62) and (63), $\mathbb{E} H_{Z_{\tau}}^{Y}(x, x+h(x)] \sim g(x)$. Further, as we have seen in the first part of the proof, for all large enough $x$,

$$
\mathbb{E} \sum_{n=\tau+\mu}^{\infty} \mathbb{I}\left\{Z_{n} \in(x, x+h(x)]\right\} \leq \varepsilon \sup _{y} H_{y}^{Z}(x, x+h(x)] .
$$

Letting $\varepsilon \rightarrow 0$ and using (64), we conclude that

$$
\mathbb{E} \sum_{n=\tau+\mu}^{\infty} \mathbb{I}\left\{Z_{n} \in(x, x+h(x)]\right\}=o(g(x)) \quad \text { as } x \rightarrow \infty .
$$

Thus, it remains to show that

$$
\mathbb{E} \mathbb{E}_{Z_{\tau}} \sum_{n=\mu}^{\infty} \mathbb{I}\left\{Y_{n} \in(x, x+h(x)]\right\}=o(g(x)) \quad \text { as } x \rightarrow \infty .
$$

But this expectation can be bounded in the same manner:

$$
\begin{aligned}
\mathbb{E}_{Z_{\tau}} \sum_{n=\mu}^{\infty} \mathbb{I}\left\{Y_{n} \in(x, x+h(x)]\right\} & \leq \mathbb{E} \mathbb{P}_{Z_{\tau}}(\mu<\infty) \sup _{y} H_{y}^{Y}(x, x+h(x)] \\
& \leq \varepsilon \sup _{y} H_{y}^{Y}(x, x+h(x)] .
\end{aligned}
$$

Combining this with (63) we complete the proof.

\section{Proofs of Theorems 1, 2 and 3}

Proof of Theorem 1. Consider a modified Markov chain $\widetilde{X}_{n}$ on the same probability space as $X_{n}$ with jumps $\widetilde{\xi}(x)$ defined as follows:

$$
\widetilde{\xi}(x)= \begin{cases}\xi(x) & \text { if }|\xi(x)| \leq s(x) \\ \text { any value } & \text { if }|\xi(x)|>s(x)\end{cases}
$$


If $\widetilde{X}_{n}$ does not satisfy the weak irreducibility condition (2), then we can increase the value of $s(x)$ on some set bounded above in such a way that then $\widetilde{X}_{n}$ do satisfy (2). Indeed, it follows from the conditions (3), (5) and (6) that there exist a sufficiently high level $x_{0}$ and an $\varepsilon>0$ such that $\mathbb{P}\{\xi(x) \geq \varepsilon\} \geq \varepsilon$ for all $x \geq x_{0}$. Then it suffices to increase $s(x)$ on the set $\left(-\infty, x_{0}\right]$ to ensure the condition (2) for $\widetilde{X}_{n}$.

Without loss of generality we assume that $h(x) \leq s(x)$. Let us choose a function $t(x) \uparrow \infty$ of order $o(h(x))$ as $x \rightarrow \infty$.

Fix some $c>1$ and consider $r(x)=c /(1+x)$. Then,

$$
R(x)=c \log (1+x) \quad \text { and } \quad U(x)=(1+x)^{1-c} /(c-1) .
$$

Therefore,

$$
U(x) e^{R(x)}=\frac{x+1}{c-1} .
$$

The chain $\widetilde{X}_{n}$ satisfies the condition (30). Fix some $c^{* *} \in(1,2 \mu / b)$ and define $r^{* *}(x)=c^{* *} /(1+x)$, which ensures the condition (31) with $\theta=\left(2 \mu / b c^{* *}-1\right) / 2>0$. The condition (29) is immediate from the upper bound

$$
\mathbb{E}\left\{|\xi(y)|^{3} ;|\xi(y)| \leq s(y)\right\} \leq s(y) m_{2}^{[s(y)]}(y)
$$

and the relation $s(y)=o(y)$. Also,

$$
m_{2}^{[t(x)]}(y) \rightarrow b \quad \text { as } x \rightarrow \infty,
$$

by the conditions (5) and (6). As a result, by Proposition 16, as $x \rightarrow \infty$,

$$
\begin{aligned}
\widetilde{H}(x+t(x), x+h(x)-t(x)] & \leq \frac{G_{h, x}^{* *}(\infty)}{b+o(1)} \\
& \leq \frac{2+o(1)}{\left(c^{* *}-1\right) b} x h(x),
\end{aligned}
$$

owing to (39) and (66). Letting $c^{* *} \rightarrow 2 \mu / b$, we get

$$
\widetilde{H}(x+t(x), x+h(x)-t(x)] \leq \frac{2+o(1)}{2 \mu-b} x h(x) \quad \text { as } x \rightarrow \infty .
$$

Taking into account that $t(x)=o(h(x))$ we conclude the following upper bound

$$
\widetilde{H}(x, x+h(x)] \leq \frac{2+o(1)}{2 \mu-b} x h(x) \quad \text { as } x \rightarrow \infty .
$$

The chain $\tilde{X}_{n}$ satisfies the condition (32). Fix some $c^{*}>2 \mu / b$ and define $r^{*}(x)=$ $c^{*} /(1+x)$, which ensures the condition (33) with $\theta=\left(1-2 \mu / b c^{*}\right) / 2>0$. Then it follows from Proposition 18 that, as $x \rightarrow \infty$,

$$
\begin{aligned}
\widetilde{H}(x-t(x), x+h(x)+t(x)] & \geq \frac{G_{h, x}^{*}(\infty)-\mathbb{E} G_{h, x}^{*}\left(X_{0}\right)-\delta(x)}{b+o(1)} \\
& \geq(2+o(1)) \frac{h(x) \frac{x}{c^{*}-1}-\delta(x)}{b+o(1)},
\end{aligned}
$$


due to (48) and (66). By the condition (5), the chain $\widetilde{X}_{n}$ satisfies (56) which together with the upper bound (68) for the renewal measure generated by $\widetilde{X}_{n}$ yields the upper bound for $\delta(x)$ delivered by Lemma 19, Therefore,

$$
\widetilde{H}(x-t(x), x+h(x)+t(x)] \geq \frac{2+o(1)}{\left(c^{*}-1\right) b} x h(x) .
$$

owing to (66). Letting here $c^{*} \rightarrow 2 \mu / b$ and since $t(x)=o(h(x))$, we finally get

$$
\widetilde{H}(x, x+h(x)] \geq \frac{2+o(1)}{2 \mu-b} x h(x) \quad \text { as } x \rightarrow \infty .
$$

Combining this lower bound with the upper bound (68), we conclude that

$$
\widetilde{H}(x, x+h(x)] \sim \frac{2}{2 \mu-b} x h(x) \quad \text { as } x \rightarrow \infty .
$$

Together with the condition (44) this allows us to apply Lemma21 to the two Markov chains, $Z_{n}=X_{n}$ and $Y_{n}=\widetilde{X}_{n}$, hence the same asymptotics for the renewal measure generated by $X_{n}$.

Proof of Theorem Q Q As in the proof of Theorem 1, from the very beginning we may assume that $|\xi(y)| \leq s(y)$ for all $y$ which implies both (30) and (32). Without loss of generality we assume that $h(x) \leq s(x)$.

Fix $c>1$ and consider

$$
\begin{aligned}
r(x)= & \frac{1}{x+e_{(m)}}+\frac{1}{\left(x+e_{(m)}\right) \log \left(x+e_{(m)}\right)} \\
& +\ldots+\frac{c}{\left(x+e_{(m)}\right) \log \left(x+e_{(m)}\right) \ldots \log _{(m)}\left(x+e_{(m)}\right)},
\end{aligned}
$$

where $e_{(m)}>0$ is defined by $\log _{(m)} e_{(m)}=1$. Therefore,

$$
\begin{aligned}
R(x)= & \log \left(x+e_{(m)}\right)+\log \log \left(x+e_{(m)}\right) \\
& +\ldots+\log _{(m)}\left(x+e_{(m)}\right)+c \log _{(m+1)}\left(x+e_{(m)}\right)-C_{m}
\end{aligned}
$$

and

$$
U(x)=\frac{e^{C_{m}}}{c-1}\left(\log _{(m)}\left(x+e_{(m)}\right)\right)^{1-c},
$$

which implies from (39) that, for $c^{* *}<\gamma+1$,

$$
G_{h(x), x}^{* *}(\infty) \leq \frac{2+o(1)}{c^{* *}-1} h(x) x \log x \ldots \log _{(m)} x \quad \text { as } x \rightarrow \infty,
$$

and from (48), for $c^{*}>\gamma+1$,

$$
G_{h(x), x}^{*}(\infty) \geq \frac{2+o(1)}{c^{*}-1} h(x) x \log x \ldots \log _{(m)} x \quad \text { as } x \rightarrow \infty .
$$

Repeating the arguments used in the proof of Theorem 1, we obtain the desired result. 
Proof of Theorem [3. As in the proof of Theorem 1, from the very beginning we may assume that $|\xi(y)| \leq s(y)$ for all $y$ which implies both (30) and (32). Without loss of generality we assume that $h(x) \leq s(x)$. Let us choose a function $t(x) \uparrow \infty$ of order $o(h(x))$ as $x \rightarrow \infty$.

Fix some $c>0$ and consider $r(x)=c v(x)$. Then, by l'Hôspital's rule,

$$
\frac{U(x)}{U^{\prime}(x)} \sim \frac{1}{r(x)} .
$$

Therefore, as follows from (39)

$$
G_{h(x), x}^{* *}(\infty) \leq(2+o(1)) \frac{h(x)}{r(x)} \quad \text { as } x \rightarrow \infty,
$$

and from (48)

$$
G_{h(x), x}^{*}(\infty) \geq(2+o(1)) \frac{h(x)}{r(x)} \quad \text { as } x \rightarrow \infty .
$$

Considering $c^{* *}<2 / b$ and $c^{*}>2 / b$ and repeating the arguments used in the proof of Theorem 1, we conclude the proof.

\section{Proof of local renewal theorem for asymptotically ho- mogeneous Markov chains}

In this section, our purpose is to provide an approach that allows us to reduce the proof of the asymptotic behaviour of the renewal measure on intervals to that on sufficiently slowly growing intervals.

Lemma 22. Assume that there exist functions $v(x)>0$ and $\widetilde{t}(x) \uparrow \infty$ such that, for any $t(x) \uparrow \infty$ satisfying $t(x) \leq \widetilde{t}(x)$,

$$
\sup _{x \geq 1} \frac{v(x) H(x, x+t(x)]}{t(x)}<\infty .
$$

Then,

$$
\sup _{x \geq 1} v(x) H(x, x+1]<\infty .
$$

Proof. Suppose that (71) fails. Then there exists a sequence $x_{n} \uparrow \infty$ such that

$$
\alpha_{n}:=v\left(x_{n}\right) H\left(x_{n}, x_{n}+1\right] \rightarrow \infty \quad \text { as } n \rightarrow \infty .
$$

Since both $\alpha_{n}$ and $\widetilde{t}\left(x_{n}\right)$ tend to infinity, there exists a sequence $t_{n} \uparrow \infty$ such that $t_{n} \leq \widetilde{t}\left(x_{n}\right)$ and $t_{n}=o\left(\alpha_{n}\right)$ as $n \rightarrow \infty$. Let $t(x)$ be defined as follows

$$
t(x)=t_{n}, \quad x_{n} \leq x<x_{n+1} .
$$

Clearly, $t(x) \leq \widetilde{t}(x)$ and $t(x) \uparrow \infty$. Then, eventually in $n$,

$$
\frac{v\left(x_{n}\right) H\left(x_{n}, x_{n}+t\left(x_{n}\right)\right]}{t\left(x_{n}\right)} \geq \frac{v\left(x_{n}\right) H\left(x_{n}, x_{n}+1\right]}{t\left(x_{n}\right)}=\frac{\alpha_{n}}{t\left(x_{n}\right)} \rightarrow \infty,
$$

which contradicts the hypothesis. 
Proof of Theorem 4. By Lemma 22 it follows from the assumption (13) that the supremum in (71) is finite. In turn, it allows us to apply Helly's Selection Theorem to the family of measures $\{v(x) H(x+\cdot), x \in \mathbb{R}\}$ (see, for example, Theorem 2 in [17, Section VIII.6]). Hence, there exists a sequence of points $x_{n} \rightarrow \infty$ such that the sequence of measures $v\left(x_{n}\right) H\left(x_{n}+\cdot\right)$ converges weakly to some measure $\lambda$ as $n \rightarrow \infty$ in the standard sense of weak convergence on bounded intervals. The following two results characterise $\lambda$.

Lemma 23. Let $F$ denote the distribution of $\xi$. A weak limit $\lambda$ of the sequence of measures $v\left(x_{n}\right) H\left(x_{n}+\cdot\right)$ satisfies the identity $\lambda=\lambda * F$.

Proof. The measure $\lambda$ is positive and $\sigma$-finite with necessity. Fix any smooth function $f(x)$ with a bounded support; let $A>0$ be such that $f(x)=0$ for $x \notin[-A, A]$. The weak convergence of measures means convergence of integrals

$$
\int_{-\infty}^{\infty} f(x) v\left(x_{n}\right) H\left(x_{n}+d x\right)=\int_{-A}^{A} f(x) v\left(x_{n}\right) H\left(x_{n}+d x\right) \rightarrow \int_{-A}^{A} f(x) \lambda(d x)
$$

as $n \rightarrow \infty$. On the other hand, due to the equality $H(\cdot)=\mathbb{P}\left\{X_{0} \in \cdot\right\}+H * P(\cdot)$ we have the following representation for the left side of (172):

$$
\int_{-A}^{A} f(x) v\left(x_{n}\right) \mathbb{P}\left\{X_{0} \in x_{n}+d x\right\}+\int_{-A}^{A} f(x) \int_{-\infty}^{\infty} P\left(x_{n}+y, x_{n}+d x\right) v\left(x_{n}\right) H\left(x_{n}+d y\right) .
$$

Since $f$ and $v$ are bounded,

$$
\int_{-A}^{A} f(x) v\left(x_{n}\right) \mathbb{P}\left\{X_{0} \in x_{n}+d x\right\} \leq\|f\|_{\infty}\|v\|_{\infty} \mathbb{P}\left\{X_{0} \in\left[x_{n}-A, x_{n}+A\right]\right\} \rightarrow 0
$$

as $n \rightarrow \infty$. The second term in (173) is equal to

$$
\int_{-\infty}^{\infty} v\left(x_{n}\right) H\left(x_{n}+d y\right) \int_{-A}^{A} f(x) P\left(x_{n}+y, x_{n}+d x\right) .
$$

The weak convergence $P(t, t+\cdot) \Rightarrow F(\cdot)$ as $t \rightarrow \infty$ implies convergence of the inner integral in (75):

$$
\int_{-A}^{A} f(x) P\left(x_{n}+y, x_{n}+d x\right) \rightarrow \int_{-A}^{A} f(x) F(d x-y)
$$

here the rate of convergence can be estimated in the following way:

$$
\begin{aligned}
\Delta(n, y) & :=\left|\int_{-A}^{A} f(x)\left(P\left(x_{n}+y, x_{n}+d x\right)-F(d x-y)\right)\right| \\
& =\left|\int_{-A}^{A} f^{\prime}(x)\left(\mathbb{P}\left\{\xi\left(x_{n}+y\right) \leq x-y\right\}-F(x-y)\right) d x\right| \\
& \leq\left\|f^{\prime}\right\|_{\infty} \int_{-A-y}^{A-y}\left|\mathbb{P}\left\{\xi\left(x_{n}+y\right) \leq x\right\}-F(x)\right| d x .
\end{aligned}
$$


Thus, the asymptotic homogeneity of the chain yields for every fixed $C>0$ the uniform convergence

$$
\sup _{y \in[-C, C]} \Delta(n, y) \rightarrow 0 \quad \text { as } n \rightarrow \infty
$$

In addition, by the majorisation condition (12), for all $x \in \mathbb{R}$,

$$
\left|\mathbb{P}\left\{\xi\left(x_{n}+y\right) \leq x\right\}-F(x)\right| \leq 2 \mathbb{P}\{\Xi>|x|\} .
$$

Hence, for all $y$,

$$
\begin{aligned}
\Delta(n, y) & \leq 2\left\|f^{\prime}\right\|_{\infty} \int_{-A-y}^{A-y} \mathbb{P}\{\Xi>|x|\} d x \\
& \leq 4 A\left\|f^{\prime}\right\|_{\infty} \mathbb{P}\{\Xi>|y|-A\} .
\end{aligned}
$$

We have an estimate

$$
\begin{aligned}
\Delta_{n} & :=\left|\int_{-\infty}^{\infty} v\left(x_{n}\right) H\left(x_{n}+d y\right)\left(\int_{-A}^{A} f(x) P\left(x_{n}+y, x_{n}+d x\right)-\int_{-A}^{A} f(x) F(d x-y)\right)\right| \\
& \leq \int_{-\infty}^{\infty} \Delta(n, y) v\left(x_{n}\right) H\left(x_{n}+d y\right) .
\end{aligned}
$$

For any fixed $C>0,(76)$ and (71) imply that

$$
\begin{aligned}
\int_{-C}^{C} \Delta(n, y) v\left(x_{n}\right) H\left(x_{n}+d y\right) & \leq \sup _{y \in[-C, C]} \Delta(n, y) \cdot \sup _{n}\left(v\left(x_{n}\right) H\left[x_{n}-C, x_{n}+C\right]\right) \\
& \rightarrow 0 \quad \text { as } n \rightarrow \infty .
\end{aligned}
$$

The remaining part of the integral can be estimated by (77):

$$
\begin{aligned}
& \limsup _{n \rightarrow \infty} \int_{|y| \geq C} \Delta(n, y) v\left(x_{n}\right) H\left(x_{n}+d y\right) \\
& \quad \leq 4 A\left\|f^{\prime}\right\|_{\infty} \limsup _{n \rightarrow \infty} \int_{|y| \geq C} \mathbb{P}\{\Xi>|y|-A\} v\left(x_{n}\right) H\left(x_{n}+d y\right) .
\end{aligned}
$$

Since $\Xi$ has finite mean, property (71) of the renewal measure $H$ allows us to choose a sufficiently large $C$ in order to make the 'lim sup' as small as we please. Therefore, $\Delta_{n} \rightarrow 0$ as $n \rightarrow \infty$. Hence, (75) has the same limit as the sequence of integrals

$$
\int_{-\infty}^{\infty} v\left(x_{n}\right) H\left(x_{n}+d y\right) \int_{-A}^{A} f(x) F(d x-y) .
$$

Now the weak convergence to $\lambda$ implies that (75) has the limit

$$
\begin{aligned}
\int_{-\infty}^{\infty} \lambda(d y) \int_{-\infty}^{\infty} f(x) F(d x-y) & =\int_{-\infty}^{\infty} f(x) \int_{-\infty}^{\infty} F(d x-y) \lambda(d y) \\
& =\int_{-\infty}^{\infty} f(x)(F * \lambda)(d x) .
\end{aligned}
$$


By (72) - (74) and (78), we conclude the identity

$$
\int_{-\infty}^{\infty} f(x) \lambda(d x)=\int_{-\infty}^{\infty} f(x)(F * \lambda)(d x)
$$

Since this identity holds for every smooth function $f$ with a bounded support, the measures $\lambda$ and $F * \lambda$ coincide. The proof is complete.

Further we use the following statement which is due to Choquet and Deny [8].

Proposition 24. Let $F$ be a distribution not concentrated at 0 . Let $\lambda$ be a nonnegative measure satisfying the equality $\lambda=\lambda * F$ and the property $\sup _{n \in \mathbb{Z}} \lambda[n, n+1]<$ $\infty$.

If $F$ is non-lattice, then $\lambda$ is proportional to the Lebesgue measure.

If $F$ is lattice with minimal span 1 and $\lambda(\mathbb{R} \backslash \mathbb{Z})=0$, then $\lambda$ is proportional to the counting measure.

The concluding part of the proof of Theorem 4 will be carried out for the non-lattice case. Choose any sequence of points $x_{n} \rightarrow \infty$ such that the measure $v\left(x_{n}\right) H\left(x_{n}+\cdot\right)$ converges weakly to some measure $\lambda$ as $n \rightarrow \infty$. It follows from Lemma 23 and Proposition 24 that then $\lambda(d x)=\alpha \cdot d x$ with some $\alpha$, i.e.,

$$
v\left(x_{n}\right) H\left(x_{n}+d x\right) \Rightarrow \alpha \cdot d x \text { as } n \rightarrow \infty .
$$

Then, for any $A>0$ and $k \in\{0,1,2, \ldots\}$,

$$
v\left(x_{n}\right) H\left(x_{n}+k A, x_{n}+(k+1) A\right] \rightarrow \alpha A .
$$

Then, there exists a sufficiently slowly growing sequence $t_{n} \uparrow \infty$ such that

$$
\frac{v\left(x_{n}\right) H\left(x_{n}, x_{n}+t_{n}\right]}{t_{n}} \rightarrow \alpha .
$$

It follows from the assumption (13) that $\alpha=C_{H}$.

We complete the proof by routine contradiction argument. Suppose there exists a sequence $\left\{x_{n}\right\}$ such that

$$
v\left(x_{n}\right) H\left(x_{n}, x_{n}+h\right] \not \rightarrow C_{H} h \quad \text { as } n \rightarrow \infty .
$$

However, by Helly's Selection Theorem and arguments above there exists a further subsequence $x_{n_{k}}$ for which

$$
v\left(x_{n_{k}}\right) H\left(x_{n_{k}}, x_{n_{k}}+h\right] \rightarrow C_{H} h,
$$

which contradicts (79). 


\section{Random walks conditioned to stay positive}

In this section we prove Example 8 by showing that under the conditions stated the random walk conditioned to stay positive satisfies all the conditions of Corollary 5 . We start with checking that there is a function $s(x) \rightarrow \infty$ of order $o(x)$ such that

$$
m_{1}^{[s(x)]} \sim \frac{\sigma^{2}}{x} \text { and } \quad m_{2}^{[s(x)]} \rightarrow \sigma^{2} \quad \text { as } x \rightarrow \infty,
$$

and (4) holds for some decreasing integrable at infinity function $p(x)$.

Indeed, it is immediate from (14) that, for all $x$ such that $x-s(x)>0$,

$$
\begin{aligned}
m_{1}^{[s(x)]}(x) & :=\frac{1}{V(x)} \mathbb{E}\left\{V\left(x+\xi_{1}\right) \xi_{1} ;\left|\xi_{1}\right| \leq s(x)\right\} \\
& =\frac{1}{V(x)} \mathbb{E}\left\{\left(V\left(x+\xi_{1}\right)-V(x)\right) \xi_{1} ;\left|\xi_{1}\right| \leq s(x)\right\}+\mathbb{E}\left\{\xi_{1} ;\left|\xi_{1}\right|>s(x)\right\} \\
& =\frac{1}{V(x)} \mathbb{E}\left\{\left(V\left(x+\xi_{1}\right)-V(x)\right) \xi_{1} ;\left|\xi_{1}\right| \leq s(x)\right\}+o(1 / x),
\end{aligned}
$$

by $\mathbb{E} \xi_{1}=0$ and the finiteness of $\mathbb{E} \xi_{1}^{2}$, provided $s(x) / x$ tends to zero sufficiently slow. Finiteness of the second moment also implies that ladder heights have finite expectation, so by the local renewal theorem,

$$
V(x+y)-V(x) \rightarrow \frac{y}{\mathbb{E} \chi^{-}} \text {as } x \rightarrow \infty,
$$

in non-lattice case; in lattice case both $x$ and $y$ are restricted to the lattice. Hence $\left(V\left(x+\xi_{1}\right)-V(x)\right) \xi_{1}$ converges a.s. to $\xi_{1}^{2} / \mathbb{E} \chi^{-}$as $x \rightarrow \infty$. By (80), $\sup _{x}(V(x+1)-$ $V(x))=: c<\infty$ which yields

$$
|V(x+y)-V(x)| \leq c_{V}(|y|+1) .
$$

This allows us to apply the dominated convergence theorem and to infer that

$$
\mathbb{E}\left\{\left(V\left(x+\xi_{1}\right)-V(x)\right) \xi_{1} ;\left|\xi_{1}\right| \leq s(x)\right\} \quad \rightarrow \quad \frac{\mathbb{E} \xi_{1}^{2}}{\mathbb{E} \chi^{-}}=\frac{\sigma^{2}}{\mathbb{E} \chi^{-}} \quad \text { as } x \rightarrow \infty .
$$

By the renewal theorem, $V(x) \sim x / \mathbb{E} \chi^{-}$and hence

$$
m_{1}^{[s(x)]}(x) \sim \frac{\sigma^{2}}{x} \quad \text { as } x \rightarrow \infty .
$$

For the truncated second moment of jumps we have

$$
\begin{aligned}
m_{2}^{[s(x)]}(x) & :=\frac{1}{V(x)} \mathbb{E}\left\{V\left(x+\xi_{1}\right) \xi_{1}^{2} ;\left|\xi_{1}\right| \leq s(x)\right\} \\
& =\frac{1}{V(x)} \mathbb{E}\left\{\left(V\left(x+\xi_{1}\right)-V(x)\right) \xi_{1}^{2} ;\left|\xi_{1}\right| \leq s(x)\right\}+\mathbb{E}\left\{\xi_{1}^{2} ;\left|\xi_{1}\right| \leq s(x)\right\} \\
& =\frac{1}{V(x)} \mathbb{E}\left\{\left(V\left(x+\xi_{1}\right)-V(x)\right) \xi_{1}^{2} ;\left|\xi_{1}\right| \leq s(x)\right\}+\sigma^{2}+o(1) .
\end{aligned}
$$


Since for $\left|\xi_{1}\right| \leq s(x)$,

$$
\left|V\left(x+\xi_{1}\right)-V(x)\right| \xi_{1}^{2} \leq c_{V}\left(1+\left|\xi_{1}\right|\right) \xi_{1}^{2} \leq c_{V}(1+s(x)) \xi_{1}^{2}
$$

so that

$$
\frac{\left|V\left(x+\xi_{1}\right)-V(x)\right|}{V(x)} \xi_{1}^{2} \quad \stackrel{\text { a.s. }}{\rightarrow} \quad 0 \quad \text { as } x \rightarrow \infty,
$$

we get, again by the dominated convergence theorem,

$$
\frac{1}{V(x)} \mathbb{E}\left\{\left(V\left(x+\xi_{1}\right)-V(x)\right) \xi_{1}^{2} ;\left|\xi_{1}\right| \leq s(x)\right\} \quad \rightarrow \quad 0 \quad \text { as } x \rightarrow \infty .
$$

Therefore,

$$
m_{2}^{[s(x)]}(x) \rightarrow \sigma^{2} \quad \text { as } x \rightarrow \infty .
$$

Summarizing, (3) holds with $\mu=\sigma^{2}$ and $b=\sigma^{2}$. According to the construction of $X_{n}$, (4) is equivalent to the following upper bound

$$
\frac{1}{V(x)} \mathbb{E}\left\{V\left(x+\xi_{1}\right) ;\left|\xi_{1}\right|>s(x)\right\} \leq \frac{p(x)}{x} .
$$

Recalling that $V(x)$ is increasing and asymptotically linear, it suffices to show that

$$
\mathbb{P}\left\{\xi_{1}<-s(x)\right\}+\frac{1}{x} \mathbb{E}\left\{\xi_{1} ; \xi_{1}>s(x)\right\} \leq \frac{p(x)}{x}
$$

for some $s(x)=o(x)$, but this is immediate from the assumption $\mathbb{E} \xi_{1}^{2}<\infty$.

We also need to check the conditions (5)-(6) and (12). To check the first one, we note that,

$$
c_{1}:=\sup _{x} \frac{V(x+s(x))}{V(x)}<\infty,
$$

hence, for $t \leq s(x)=o(x)$,

$$
\begin{aligned}
\mathbb{P}\{|\xi(x)|>t,|\xi(x)| \leq s(x)\} & =\left(\int_{-s(x)}^{-t}+\int_{t}^{s(x)}\right) \frac{V(x+u)}{V(x)} \mathbb{P}\left\{\xi_{1} \in d u\right\} \\
& \leq c_{1} \mathbb{P}\left\{\left|\xi_{1}\right|>t\right\}
\end{aligned}
$$

and (5)-(6) follows if we take $\widehat{\xi}$ defined by its tail as

$$
\mathbb{P}\{\widehat{\xi}>t\}=\min \left\{1, c_{1} \mathbb{P}\left\{\left|\xi_{1}\right|>t\right\}\right\},
$$

which is square integrable because $\xi_{1}$ is so.

Next, using once again (81) we obtain

$$
\begin{aligned}
\mathbb{P}\{|\xi(x)|>t\} & =\left(\int_{-x}^{-t}+\int_{t}^{\infty}\right) \frac{V(x+u)}{V(x)} \mathbb{P}\left\{\xi_{1} \in d u\right\} \\
& \leq \mathbb{P}\left\{\xi_{1}<-t\right\}+\int_{t}^{\infty}\left(1+c_{V} \frac{u+1}{V(x)}\right) \mathbb{P}\left\{\xi_{1} \in d u\right\} \\
& \left.\leq \mathbb{P}\left\{\xi_{1}<-t\right\}+\left(1+\frac{c_{V}}{V(x)}\right) \mathbb{P}\left\{\xi_{1}>t\right\}+\frac{c_{V}}{V(x)} \mathbb{E}\left\{\left|\xi_{1}\right| ;\left|\xi_{1}\right|>t\right\}\right) \\
& \leq c_{2}\left(\mathbb{P}\left\{\left|\xi_{1}\right|>t\right\}+\mathbb{E}\left\{\left|\xi_{1}\right| ;\left|\xi_{1}\right|>t\right\}\right) \text { for all } x, t>0 .
\end{aligned}
$$


The right hand side is integrable due to $\mathbb{E} \xi_{1}^{2}<\infty$, so the condition (12) is satisfied too.

Finally, the asymptotic homogeneity (10) is immediate from (14), with $\xi=\xi_{1}$, because, for any fixed $u \in \mathbb{R}, V(x+u) / V(x) \rightarrow 1$ as $x \rightarrow \infty$, and the proof is complete.

\section{References}

[1] Alexander, K. S. (2011) Excursions and local limit theorems for Bessel-like random walks. Electron. J. Probab. 16, 1-44.

[2] Athreya, K. B., McDonald, D. and Ney, P. (1978) Limit Theorems for SemiMarkov Processes and Renewal Theory for Markov Chains. Ann. Probab. 6, 788-797.

[3] Berger, Q. (2019) Strong renewal theorems and local large deviations for multivariate random walks and renewals. Electron. J. Probab. 24, 47pp.

[4] Bertoin, J. and Doney, R. A. (1994) On conditioning a random walk to stay nonnegative. Ann. Probab. 22, 2152-2167.

[5] Blackwell, D. (1948) A renewal theorem. Duke Math. J. 15, 145-150.

[6] Blackwell, D. (1953) Extension of a renewal theorem. Pacific J. Math. 3, 315320.

[7] Caravenna, F. and Doney, R.(2019) Local large deviations and the strong renewal theorem. Electron. J. Probab. 24, paper no. 72, 48 pp.

[8] Choquet, G. and Deny, J. (1960) Sur l'équation de convolution $\mu=\mu * \sigma$. C. R. Acad. Sci. Paris Série A, 250, 799-801.

[9] Cox, D. R. and Smith, W. L. (1953) A direct proof of a fundamental theorem of renewal theory. Scand. Actuarial J. 36, 139-150.

[10] Denisov, D. E. (2006) On the existence of a regularly varying majorant of an integrable monotone function. Math. Notes 76, 129-133.

[11] Denisov, D., Korshunov, D. and Wachtel, V. (2013) Potential analysis for positive recurrent Markov chains with asymptotically zero drift: Power-type asymptotics. Stoch. Process. Appl. 123, 3027-3051.

[12] Doney, R. A. (1966). An analogue of the renewal theorem in higher dimensions. Proc. London Math. Soc. 16(3), 669-684.

[13] Erdös, P., Feller, W. and Pollard, H. (1949) A property of power series with positive coefficients. Bull. Amer. Math. Soc. 55, 201-204.

[14] Erickson, K. B. (1970) Strong renewal theorems with infinite mean. Trans. Amer. Math. Soc. 151, 263-291. 
[15] Feller, W. (1941) On the Integral Equation of Renewal Theory. Ann. Math. Statist. 12, 243-267.

[16] Feller, W. and Orey, S. (1961) A renewal theorem. J. Math. Mech. 10, 619-624.

[17] Feller, W. (1971) An Introduction to Probability Theory and Its Applications Vol. 2, Wiley, New York.

[18] Garsia, A. and Lamperti, J. (1962). A discrete renewal theorem with infinite mean. Comment. Math. Helv. 37, 221-234.

[19] Guibourg, Denis; Hervé, Loic (2013). Multidimensional renewal theory in the non-centered case. Application to strongly ergodic Markov chains. Potential Anal. 38 (2), 471-497.

[20] Guivarc'h, Y., Keane, M., and Roynette, B. (1977) Marches aléatoires sur les groupes de Lie. (French) Lecture Notes in Mathematics, Vol. 624. SpringerVerlag, Berlin-New York.

[21] Kesten, H. (1974) Renewal theory for functionals of a Markov chain with general state space. Ann. Probab. 2, 355-386.

[22] Klüppelberg, C. and Pergamenchtchikov, S. (2003) Renewal theory for functionals of a Markov chain with compact state space. Ann. Probab. 31, 2270-2300.

[23] Korshunov, D. (2008) The key renewal theorem for a transient Markov chain. J. Theor. Probab. 21, 234-245.

[24] Lamperti, J. (1960) Criteria for the recurrence or transience of stochastic processes I. J. Math. Anal. Appl. 1, 314-330.

[25] Lamperti, J. (1962) A new class of probability limit theorems. J. Math. Mech. 11, 749-772.

[26] Lamperti, J. (1963) Criteria for stochastic processes II: passage time moments. J. Math. Anal. Appl. 7, 127-145.

[27] Maejima, M. (1975) On local limit theorems and Blackwell's renewal theorem for independent random variables. Ann. Inst. Stat. Math. 27, 507-520.

[28] Mellein, B. (1983) Green function behaviour of critical Galton-Watson process with immigration. Bol. Soc. Brasil. Mat. 14, 17-25.

[29] Menshikov, M. V., Asymont, I. M., and Yasnogorodskii, R. (1995) Markov processes with asymptotically zero drifts. Probl. Inform. Trans. 31, 248-261.

[30] Menshikov, M., Popov, S., and Wade, A. (2017) Non-homogeneous Random Walks. Lyapunov Function Methods for Near-Critical Stochastic Systems. Cambridge University Press. 
[31] Meyn, S. P., and Tweedie, R. L. (1993). Markov Chains and Stochastic Stability. Springer-Verlag, London.

[32] Nagaev, A. V. (1980) Renewal Theorems in $R^{d}$. Theory Probab. Appl., 24(3), $572-581$.

[33] Pakes, A.G. (1972) Further results on the critical Galton-Watson process with immigration. J. Austral. Math. Soc. 13, 277-290.

[34] Port, S. C. and Stone, C. J. (1967) Hitting time and hitting places for nonlattice recurrent random walks. J. Math. Mech. 17, 35-57.

[35] Revuz, D. and Yor, M. (1999) Continuous Martingales and Brownian Motion, 3rd Edition. Springer-Verlag, Berlin.

[36] Rogozin, B. (1977) Asymptotics of renewal functions. Theory Probab. Appl. 21, 669-686.

[37] Rosenkrantz, W. A. (1966) A local limit theorem for a certain class of random walks. Ann. Math. Statist. 37, 855-859.

[38] Smith, W. L. (1961) On some general renewal theorems for nonidentically distributed variables. Proc. 4th Berkeley Sympos. Math. Statist. and Prob., Vol. 2: Contributions to Probability Theory, 467-514.

[39] Spitzer, F. (1964) Principles of Random walks. Prinston New Jersey: Toronto.

[40] Shurenkov, V. (1984) On Markov renewal theory. Theory Probab. Appl. 29, 247-265.

[41] Williamson, J. A. (1965) Some renewal theorems for non-negative independent random variables. Trans. Amer. Math. Soc. 114, 417-445. 